The abstracts are only available online, free of charge, at www.karger.com/doi/10.1159/000201719

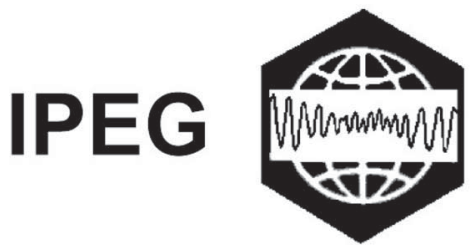

International Pharmaco-EEG Society

Association for Electrophysiological Brain Research in Preclinical and Clinical Pharmacology and Related Fields

\title{
15th Biennial Meeting of the International Pharmaco-EEG Group (IPEG)
}

FORENAP - Rouffach, France, September 24-27, 2008

\section{Abstracts}

President of the IPEG and of the Scientific Program Committee

Gé S.F. Ruigt, Oss (The Netherlands)

President of the Local Organizing Committee

Rémy Luthringer, Rouffach (France) 


\section{Neuropsychobiology}

\section{Contents}

Symposia

Session 1

The Place of EEG Studies in Animals

Session 2

Application of Q-EEG to Drug Evaluation in Neurology

Session 3

Application of Q-EEG to Drug Evaluation in Psychiatry

Session 4

Sleep EEG in Translational Research from Neuroscience to Clinical Psychiatry

Session 5

Relevance of Pharmaco-EEG: A Debate Between Academia and Industry

Session 6

EEG-Based Personalized Medicine

Session 7

EEG Studies in the Ketamine/NMDA Model of Cognitive Impairments and

Schizophrenia

Session 8

Complementary Techniques Applied to Drug Neurophysiological Research in Healthy Volunteers

Posters

Free Oral Communications 


\section{Session 1: The Place of EEG Studies in Animals}

\author{
S1.1 \\ Pharmacological Characterisation of the Sleep-Wake \\ Effects of the Antidepressant Mirtazapine \\ N. Ward ${ }^{1}$, G.S.F. Ruigt ${ }^{2}$, P.C.M. Vijn ${ }^{2}$, K. Anderson ${ }^{1}$, C. Elands ${ }^{1}$, \\ H.M. Marston ${ }^{1}$ \\ ${ }^{1}$ Organon Laboratories Ltd a part of the Schering-Plough \\ Corporation, Newhouse, ML1 5SH, Scotland, UK; ${ }^{2}$ Organon N.V. \\ a part of the Schering-Plough Corporation, Oss, The \\ Netherlands
}

Introduction: Mirtazapine is an atypical antidepressant that unlike re-uptake inhibitors (e.g., SSRIs) influences monoaminergic neurotransmission by blocking $\mathrm{a}_{2}, 5-\mathrm{HT}_{2}, 5-\mathrm{HT}_{3}, 5-\mathrm{HT}_{6} \&$ $5-\mathrm{HT}_{7}$ receptors. The preclinical \& clinical profile of mirtazapine also differs from drugs such as SSRIs, with significant sleep improving properties observed for mirtazapine. These observations were extended by probing the pharmacological properties of mirtazapine in a series of studies in the rat using the ACSO (Automatic Classification of Sleep Organization) system to quantify sleep-wake. Method: Sleep-waking behaviour of male Sprague-Dawley rats was automatically classified on the basis of EEG, EMG \& Movement scores for each 2s epoch into 6 classes: active waking, passive waking, light sleep, deep sleep, intermediate sleep \& REM sleep. A 14.5 hour experimental registration period was recorded following either vehicle(s) or dose(s) of the drug. Results: Consistent with previous findings mirtazapine increased deep sleep \& inhibited REM sleep in the rat. The wake inducing effects of the 5- $\mathrm{HT}_{2}$ agonist DOI \& the $\mathrm{H}_{3}$ antagonist ciproxifan could be reversed by mirtazapine pre-treatment at doses that increased deep sleep. The reversal of DOI \& ciproxifan were characterised using selective $5-\mathrm{HT}_{2} \& \mathrm{H}_{1}$ antagonists. The inhibition of REM sleep by mirtazapine could also be reversed by the $5-\mathrm{HT}_{1 \mathrm{~A}}$ antagonist WAY100635. Conclusion: The ACSO system was successfully used to identify the atypical antidepressant mirtazapine, highlighting several properties including potential sleep modifying (deep sleep increase) \& antidepressant-like properties (REM inhibition). In addition 5- $\mathrm{HT}_{2 \mathrm{~A}} \& \mathrm{H}_{1}$ antagonist effects were observed at a dose that increased deep sleep in the rat. The inhibition of REM sleep by mirtazapine could be blocked by a $5-\mathrm{HT}_{1 \mathrm{~A}}$ antagonist thereby implicating an increase in $5-\mathrm{HT}_{1 \mathrm{~A}}$ neurotransmission in the mechanism of action of mirtazapine.

\section{S1.2 \\ Quantitative Pharmaco-Electroencephalography in
Drug Discovery: An Old Friend Revisited}

\section{A. Ahnaou, W.H.I.M. Drinkenburg}

Dept. of Neuroscience, RED Europe Johnson \& Johnson PRD, Beerse, Belgium

Sleep and pharmacoEEG are important complementary tools for investigating the temporal dynamics of brain neuronal activities, but also offer unique opportunities for combination with more recently introduced techniques in neuroscience. The added value and relevance of pharmacoEEG to preclinical evaluation of psychotropic drugs will in detail be discussed in a case study, in which the efficacy on sleep architecture and EEG of a new wakepromoting agent was compared to widely prescribed CNS psychostimulant reference drugs in different rodent species. Integration of pEEG findings with molecular pharmacogenetics provided a rationale for the unique wake-promoting efficacy of the test compound and suggested a potentially valuable therapy of vigilance deficiency and sleep-wake disorders such as excessive daytime sleepiness. The case study clearly illustrates the persisting value of pEEG and sleep-wake analysis in rodents also when applied in a more integrative framework of drug discovery and development research.

\section{S1.3 \\ Disturbed Sleep in MPTP-Induced Parkinsonian Marmoset Monkeys \\ I.H.C.H.M. Philippens, P.S. Verhave, R.A.P. Vanwersch \\ BPRC, Lange Kleiweg 139, Rijswijk, The Netherlands}

The increasing prevalence of neurodegenerative diseases, like Parkinson's disease (PD), is an increasing concern for the ageing western societies. As the current medications cannot cure the progressive loss of neurons, neuroprotective strategies should be considered. However, when PD is diagnosed already $60 \%$ of the dopaminergic neurons are lost. Therefore early biomarkers are

\section{KARGER \\ Fax +41613061234 \\ E-Mail karger@karger.ch}

www.karger.com (c) 2009 S. Karger AG, Basel

$0302-282 X / 08 / 0584-0223 \$ 24.50 / 0$

Accessible online at:

www.karger.com/nps 
needed for early detection of signs and subsequent early intervention in the accumulating neuronal loss. Sleep dysfunction and sleep disorders are common in patients with neurodegenerative disease. Sleep abnormalities such as disordered REM sleep with enhanced muscle tone is found in patients with $\mathrm{PD}$, and may precede the clinically evident symptoms of PD by years. Therefore, biomarkers on sleep disturbances may be a useful tool for early detection. To examine the effects of neurodegeneration on sleep pattern the nocturnal sleep of 1-methyl, 4-phenyl, 1,2,3,6- tetrahydropyridine (MPTP) induced parkinsonian marmoset monkeys was telemetrically registered. Two electroencephalogram (EEG) electrodes and two electromyogram (EMG) electrodes were placed and connected with a transmitter. Telemetric registration of the EEG and EMG was performed before and several days after subchronic MPTP treatment, the preferential counterpart of idiopathic PD. A total dose of $7 \mathrm{mg} / \mathrm{kg}$ MPTP was given within a two-week period. Besides sleep disturbances, an increase of parkinsonian symptoms and motor deterioration was observed. As sleep is similarly affected in PD animal models such as a decreased amount of rapid eye movement (REM) and slow wave sleep, and the MPTP model is clearly linked to a form of human parkinsonism that is indistinguishable from idiopathic PD, the MPTP marmoset monkey is the preferential model for measuring sleep dysfunction in PD research.

\section{S1.4 \\ Compounds Interacting Selectively with $\mathrm{GABA}_{\mathrm{Aa} 2}$ Subunits Elicit Distinct and Potentially Translatable Spontaneous and Induced EEG Spectral Modulations in the Behaving Rat}

E.P. Christian, D.H. Snyder, W. Song, B. Potts, J.J. Doherty, M.C. Quirk

Department of Neuroscience; AstraZeneca Pharmaceuticals, Wilmington, Del., USA

Cortical neuronal networks generate oscillatory firing that critically depends on GABAergic inhibition, and relates to ongoing behavior. This synchronized activity is detected over a series of EEG frequency bands $(\delta: 1-5, \theta: 6-8, \alpha: 9-12, \beta: 13-30, \gamma: 31-50$ $\mathrm{Hz}$ ) emanating from different circuits and generated by distinct physiological mechanisms. Benzodiazepine drugs interact nonselectively with $\mathrm{GABA}_{\mathrm{A \alpha 1}, 2 / 3,5}$ subunits and produce a complex modulatory signature across multiple EEG bands (i.e., increased $\delta, \beta, \gamma$ and decreased $\alpha, \theta$ power) that is associated with sedation, and is translatable between preclinical species and man. Recently, drug development has focused on $\mathrm{GABA}_{\mathrm{Aa}}$ subtype selective compounds in an effort to dissociate the nearly overlapping anxiolytic and sedative profiles of benzodiazepines. Here we compared in rats the effects of the benzodiazepine, Lorazepam, the al-selective drug, Zolpidem, and the $\alpha 2 / 3$-selective development compounds, TPA003 and TPA023 on the spontaneous EEG spectral signature, and $\beta / \gamma$ induced EEG components following a tone conditioned stimulus during an auditory operant task. Zolpidem and $\alpha 2 / 3$ selective compounds differentiated by their capacity to increase, respectively, low frequency a vs. high frequency $\gamma$ power in a dosedependent manner. The Lorazepam signature encompassed all of these effects. The effects on spontaneous EEG bands were paralleled by differential modulation of event-related synchronization and desynchronization of $\beta / \gamma$ oscillations across time envelopes associated with sensory processing, motor initiation, and reward expectancy in the operant task. These differences may reflect mechanisitic consequences of regional and subcellular localization of the targeted $\mathrm{GABA}_{\mathrm{A \alpha}}$ subunits within the brain, and may provide effective translatable biomarkers for developing $\mathrm{GABA}_{\mathrm{A} \alpha}$ selective compounds.

\section{Session 2: Application of Q-EEG to Drug Evaluation in Neurology}

\author{
S2.1 \\ Mood Disturbance and Nogo ERPs in Parkinson's \\ Disease (PD)
}

\section{Osawa}

Department of Neurology, Neurological Institute, Tokyo Women's Medical University, Tokyo, Japan

Mood disorders in PD comprise depressive state, apathy, anhedonia, central fatigue, a symptom related with depressive state, etc. Their responsible lesions are thought to be in the mesocorti$\mathrm{cal} /$ mesolimbic dopaminergic system, especially the orbitofrontal lobe and/or the anterior cingulate cortex. These regions are also thought to be the generator sources of event-related potentials (ERPs) evoked by nogo stimuli, i.e. nogo N2 and nogo P3. The subjects included $33 \mathrm{PD}$ patients at an early stage without dementia and 53 age-matched patient controls. Depression scores of selfrating depression scale (SDS) (Zung, 1965) and also fatigue scores evaluated with Brown's Questionnaire (2005) were significantly $(\mathrm{P}<0.05)$ greater in $\mathrm{PD}$ patients than in controls. Scores of categories achieved in the Wisconsin Card Sorting Test (WCST) and scores of go-no go paradigm in the Frontal Assessment Battery (FAB) (Dubois, et al, 2000) were significantly $(\mathrm{P}<0.05, \mathrm{P}<0.01$, respectively) lower in $\mathrm{PD}$ patients than in controls. The former is reported to reflect functions of the dorsolateral prefrontal cortex: on the other hand, the latter is reported to be related with functions of the orbitofrontal lobe and/or the anterior cingulate cortex. Neither test scores were significantly correlated with the Unified Parkinson's Disease Rating Scale scores. Nogo N2 amplitude showed tendency to be larger in PD patients than in controls. At $\mathrm{Fz}$ and $\mathrm{Cz}$, it correlated significantly with apathy scores but not with SDS scores. In conclusion, nogo ERPs in addition to mood scales and psychometric tests might be useful for evaluation of ant-PD drugs especially in regards to functions of the frontal lobes including the orbitofrontal cortex and the anterior cingulate cortex as well as the dorsolateral prefrontal cortex. 


\section{S2.2}

\section{Cholinergic Systems and Cortical Rhythms in Alzheimer Disease: New Findings}

C. Babiloni ${ }^{1,2,3}$, B. Frisoni Giovanni ${ }^{4}$, M. Pievani ${ }^{4}$, C. Del Percio ${ }^{4}$, F. Vecchio ${ }^{3,4}$, C. Geroldi ${ }^{4}$, F. Eusebi ${ }^{5,6}$, C. De Carli ${ }^{7}$, P.M. Rossini $i^{2,3,8}$

${ }^{1}$ Department of Biomedical Sciences, University of Foggia, Italy; ${ }^{2}$ San Raffele Cassini and IRCCS San Raffaele Pisana, Rome, Italy; ${ }^{3}$ A.Fa.R., Dip. Neurosci. Osp. FBF; Isola Tiberina, Rome, Italy; ${ }^{4}$ IRCCS "S. Giovanni di Dio-F.B.F.", Brescia, Italy; ${ }^{5}$

Department of Physiology and Pharmacology, University of Rome Sapienza, Rome, Italy; ${ }^{6}$ IRCCS "Neuromed", Pozzilli (IS), Italy; ${ }^{7}$ Department of Neurology and Imaging of Dementia and Aging Laboratory, Center for Neuroscience, University of California at Davis, Sacramento, Calif., USA; ${ }^{8}$ Clin. Neurol. University "Campus Biomedico", Rome, Italy

Acetylcholinesterase inhibitors (AChEI) such as donepezil act in mild Alzheimer's disease (AD) by increasing cholinergic tone. Differences in the clinical response in patients who do or do not benefit from therapy may be due to different functional features of the central neural systems. We tested this hypothesis using cortical electroencephalographic (EEG) rhythmicity. Resting eyes closed EEG data were recorded in 58 mild AD patients (Mini Mental State Examination [MMSE] range 17- 24) before and approximately 1 year after standard donepezil treatment. Based on changes of MMSE scores between baseline and follow-up, 28 patients were classified as "Responders" (MMSEvar_0) and 30 patients as "Non-Responders" (MMSEvar $<0)$. EEG rhythms of interest were delta $(2-4 \mathrm{~Hz})$, theta $(4-8 \mathrm{~Hz})$, alpha $1(8-10.5 \mathrm{~Hz})$, alpha $2(10.5-13 \mathrm{~Hz})$, beta $1(13-20 \mathrm{~Hz})$, and beta $2(20-30 \mathrm{~Hz})$. Cortical EEG sources were studied with low-resolution brain electromagnetic tomography (LORETA). Before treatment, posterior sources of delta, alpha 1 and alpha 2 frequencies were greater in amplitude in Non-Responders. After treatment, a lesser magnitude reduction of occipital and temporal alpha 1 sources characterized Responders. These results suggest that Responders and Non-Responders had different EEG cortical rhythms. Donepezil could act by reactivating existing yet functionally silent cortical synapses in Responders, restoring temporal and occipital alpha rhythms. In this line, we tested the hypothesis that alpha rhythms are affected at preclinical stage of $\mathrm{AD}$, namely amnesic mild cognitive impairment (aMCI). The aMCI patients were divided into two groups of high (MCI Ch+; N=29; $\mathrm{MMSE}=26.2$ ) and low cholinergic damage (MCI Ch-; N=28; MMSE=26.6). Power of occipital, parietal, temporal, and limbic alpha 1 sources was maximum in Nold, intermediate in MCI Ch-, and low in MCI Ch+ patients, confirming the hypothesis.

\section{S2.3}

sLORETA-Based Age Dependent Norms of Default Mode Electric Neuronal Activity: Validation in Acute Cefrebral Cortical Infarction Confirmed By SPECT and MRI

\author{
R.D. Pascual-Marqui ${ }^{1}$, H. Tanaka ${ }^{2}$, Y. Hoshino ${ }^{2}$, K. Hirata ${ }^{2}$, \\ E.R. John ${ }^{3}$, L. Prichep ${ }^{3}$, R. Isenhart ${ }^{3}$
}

${ }^{1}$ The KEY Institute for Brain-Mind Research, University Hospital of Psychiatry, Zurich, Switzerland; ${ }^{2}$ Department of Neurology, Dokkyo Medical University, Tochigi, Japan; ${ }^{3}$ Brain Research Laboratories, Department of Psychiatry, NY University School of Medicine, New York, N.Y., USA

Developmental equations were computed for brain electric activity in the age range 17-80 years, based on 139 normal controls from the NYU Brain Research Labs database. EEG was recorded from 19 electrodes (10/20 placement system) under awake, resting, eyes closed conditions, i.e. in the default mode. As a second step, signals of electric neuronal activity were estimated at 6239 virtually implanted electrodes. This was achieved by applying sLORETA (standardized low resolution brain electromagnetic tomography) to the EEG recordings, thus computing post-synaptic current density at 6239 voxels ( $5 \mathrm{~mm}$ resolution) distributed on the cortex. These computed signals were then used for estimating the spectral densities on the cortex, in the $1.5-35 \mathrm{~Hz}$ range with $0.39 \mathrm{~Hz}$ frequency resolution. Simple parametric age regression equations were computed under several scalings: absolute spectra, relative spectra, subject-wise scaling, and frequency-wise scaling. In all cases approximate Gaussianity of the residuals was guaranteed by using the logarithm of age, logarithm of absolute power, and the z-Fisher transform for relative power. Orderly and smooth spatio-frequency changes with age were observed for the absolute power spectra: theta power decreased with age in central regions, alpha power decreased with age in parieto-occipital regions, beta power increased with age in fronto-temporal regions, and gamma power decreased with age in parietal regions. Default mode EEG and SPECT were recorded on the same day in 9 patients with acute cortical infarction within one month after stroke onset. Significant deviations from normal controls were observed in different aspects of the default mode activity. In 6 cases significant abnormal relative power excess of slow activity was localized at the boundaries of the infracted regions; in 6 cases significant abnormal power excess (with subject-wise scaling) of slow activity was localized correctly; in 4 cases significant abnormal decreased power (with frequency-wise scaling) of alpha activity was localized correctly; and in 5 cases significant abnormal decreased power (with frequency-wise scaling) of beta activity was localized correctly. These results are very encouraging, supporting the use of the BRL-sLORETA norms in neurological practice. 


\section{S2.4}

Decrease in Propagation of Interictal Epileptiform Activity After Introduction of Levetiracetam, Visualized with Electric Source Imaging

\section{G. Lantz ${ }^{1,2}$, C.M. Michel ${ }^{2}$, M. Seeck ${ }^{3}$, G. Larsson Pål}

${ }^{1}$ The National Centre for Epilepsy, Sandvika, Norway;

${ }^{2}$ Functional Brain Mapping Laboratory, University of Geneva;

${ }^{3}$ Neurology Clinic, University Hospital, Geneva, Switzerland

Purpose: Different neuroimaging techniques (fMRI, Spectroscopy, PET) are being used to evaluate candidate drugs in pharmacological development. In patients with epilepsy fast propagation of the epileptiform activity between different brain areas may occur. Electric Source Imaging (ESI), in contrast to the aforementioned techniques, has a millisecond time resolution, allowing visualization of this fast propagation. The purpose of the current project was to use ESI to investigate whether introduction of an antiepileptic drug (levetiracetam, LEV) would change the propagation patterns of the interictal epileptiform activity. Methods: Twenty-four patients with epilepsy were subject to an EEG recording before (Pre-LEV) and after (In-LEV) introduction of LEV. Interictal spikes with similar topographic distribution were averaged within each subject, and a distributed source model was used to localize the EEG sources of the epileptiform activity. The temporal development of the activity within 40 regions of interest (ROIs) was determined, and source propagation between different regions was compared between the Pre-LEV and In-LEV recordings. Results: Patients with epileptic seizures showed propagation in 18/18 identified spike types in the Pre-LEV recordings. In the In-LEV recordings only $4 / 11$ spike types showed propagation, and three of these four propagating spikes were recorded in patients with poor effect of treatment. Also in patients without seizures LEV tended to suppress propagation. Discussion: We conclude that the observed suppression of source propagation can be considered as an indicator of effective antiepileptic treatment. ESI might thus become a useful tool in the early clinical evaluation of new candidate drugs in pharmacological development.

\section{Session 3: Application of Q-EEG to Drug Evaluation in Psychiatry}

\section{S3.1 \\ Polysomnographic and Q-EEG Studies in Young and Elderly Normal Subjects Under First- and Second- Generation Antipsychotics}

\author{
B. Saletu, P. Anderer, J. Stanek, R. Nikzad, A. Tajmir-Riahi, \\ N. Pintzinger, G.M. Saletu-Zyhlarz
}

Department of Psychiatry and Psychotherapy, Medical University of Vienna, Austria

In the treatment of nonorganic insomnia the $1^{\text {st }}$ and $2^{\text {nd }}$ generation antipsychotics prothipendyl (P) and quetiapine (Q), respectively, are frequently used, but controlled sleep and awakening studies, specifically in different age groups, are lacking. Nine young and 9 elderly normal volunteers spent 5 nights in the sleep laboratory (adaptation, baseline, randomized placebo, $50 \mathrm{mg} \mathrm{Q}$ and $80 \mathrm{mg} \mathrm{P}$ nights). Objective and subjective sleep and awakening quality was measured by polysomnography and psychometry. A 3-min V-EEG (10 h post evening drug at 21.00) was analyzed by EEG-mapping and low-resolution brain electromagnetic tomography (LORETA). Regarding sleep initiation and maintenance, in young subjects $\mathrm{Q}$ improved sleep latency, total sleep time and nocturnal awakenings, while $\mathrm{P}$ showed no changes as compared with placebo. In elderlies, both drugs improved all variables, with the placebo-corrected differences being more pronounced than in young subjects. Further differential effects were observed in sleep architecture, snoring, respiration, periodic leg movements and subjective sleep and awakening quality. As demonstrated by EEGLORETA, Q induced a theta increase in the right dorsolateral and ventrolateral prefrontal cortex, an alpha-2 increase right more than left frontally as well as a beta decrease in the right parietal and temporal lobe, in bilateral occipital regions and the limbic system. $\mathrm{P}$ reduced delta activity left temporally, occipitally and in the limbic system, increased alpha-2 activity right more than left frontally, in the limbic system and right temporally as well as beta-3 activity bilaterally in the medial PFC. Thus, Q induced morning EEG changes typical of sedative antipsychotics, $P$ changes indicative of regulatory CNS effects after a short half-life drug. 
S3.2

\section{Frontal QEEG-Guided Antidepressant Selection May Improve Response and Remission Rates: Insights From The BRITE-MD Trial}

\author{
I.A.Cook ${ }^{1}$, A.F. Leuchter ${ }^{1}$, W.S. Gilmer' ${ }^{2}$, S.D. Greenwald ${ }^{3}$, \\ R.H. Howland ${ }^{4}$, M.H. Trivedi ${ }^{5}$ \\ ${ }^{1}$ UCLA Laboratory of Brain, Behavior, and Pharmacology, Semel \\ Institute, Los Angeles, Calif., ${ }^{2}$ Psychiatry, Northwestern \\ University, Chicago, III., ${ }^{3}$ Neuroscience, Aspect Medical Systems, \\ Norwood, Mass., ${ }^{4}$ Western Psychiatric Institute and Clinic, \\ University of Pittsburgh, Pittsburgh, Pa., ${ }^{5}$ Psychiatry, University \\ of Texas Southwestern Medical School, Dallas, Tex., USA
}

Objective: The BRITE-MD study (www.BRITE-MD.com , NCT00289523) assessed the accuracy of a frontal quantitative EEG (fqEEG) biomarker in predicting treatment outcome with escitalopram (ESC). This analysis compares response and remission rates between subjects receiving treatment consistent with the biomarker prediction vs. other subjects. Methods: A total of 375 adults with DSM-IV-defined Major Depressive Disorder (MDD) enrolled in the trial. Subjects began treatment with ESC (10 mg/ day), and after 1 week were randomized either to: 1) continue ESC (10 mg/d) for 7 more weeks; 2) switch to bupropion XL (BUP; $300 \mathrm{mg} / \mathrm{d}$ ) for 7 weeks; or, 3) augment with bupropion XL (AUG; $300 \mathrm{mg} / \mathrm{d}$ ). Symptom severity was assessed with the Hamilton Depression Rating Scale (HAM-D-17) and 4-channel fqEEG was recorded. Outcomes were response $(>=50 \%$ decrease in HAMD) and remission (final HAM-D $<=7$ ). A composite EEG index (Antidepressant Treatment Response, ATR rev 4.1) was developed to predict clinical response using fqEEG from baseline to week 1. Both subjects and research staff were blinded to fqEEG status. Results: 220 subjects (age 43 (s.d. 13) years; $62 \%$ female) were evaluated, after excluding protocol violators and subjects with EEG artifact; ESC n=73, BUP $n=73$, AUG $n=74$. For subjects remaining on ESC, the response rate was significantly higher in ATRpredicted responders than for ATR-predicted non-responders ( $68 \%$ vs. $28 \%, p=0.001$ ). Similarly, for subjects remaining on ESC, the remission rate was significantly higher in the ATR-predicted remitters than the ATR-predicted non-remitters (50\% vs. $21 \%$, $\mathrm{p}=0.010)$. ATR-predicted non-responders who were randomized to BUP had a significantly higher response rate compared to those remaining on ESC ( $53 \%$ vs. $28 \%, \mathrm{p}=0.034$ ). ATR-predicted nonresponders who were augmented had a modestly higher response rate compared to those remaining on ESC treatment (33\% vs. $28 \%$, $\mathrm{p}=\mathrm{ns}$.) Conclusions: The use of a fqEEG biomarker at week 1 of ESC treatment may help guide antidepressant selection. Subjects whose ATR predicts response or remission do better when continued on ESC, while subjects whose ATR predicts poorer outcomes may benefit from alternate regimens.

\section{S3.3}

\section{EEG Synchronization in Untreated Schizophrenia}

T. Koenig ${ }^{1}$, M. Kikuchi ${ }^{3}$, M. Yoshimura ${ }^{2}$, D. Lehmann ${ }^{4}$, T. Dierks ${ }^{1}$, W. Strik

${ }^{1}$ Department of Psychiatric Neurophysiology, University Hospital of Clinical Psychiatry, Bern; Switzerland; ${ }^{2}$ Department of Neuropsychiatry, Kansai Medical University, Osaka, Japan; ${ }^{3}$ Department of Psychiatry and Neurobiology, Graduate School of Medical Science, Kanazawa University,Kanazawa, Japan; ${ }^{4}$ The KEY Institute for Brain-Mind Research, University Hospital of Psychiatry, Zurich, Switzerland

Background: Higher order cognition integrates information across large-scale neurocognitive networks. This integration is assumingly achieved by synchronizing neural activity within the network (binding). Such networks form not only during task execution, but also spontaneously, defining the subject's current state of mind. The interaction of pre-activated networks with activity elicited by an external stimulus determines the interpretation of the stimulus and eventually the selection of a behavioral response. Abnormal internal states may therefore be priors to abnormal perceptions and behavior observed in schizophrenics. Methods: Spontaneous brain activity can be measured by EEG, and the relation between different EEG channels contains information about the synchronization of brain processes. We pool a series of studies we have conducted, investigating EEG synchronization in untreated schizophrenic patients and healthy controls. Dependent variables were the duration, occurrence and spatial distribution of transiently synchronized EEG states (so called microstates) and a frequency-domain measure of phase communality across electrodes (GFS). Results: Across all studies, schizophrenics showed reduced duration of a defined subset of microstates, which suggests premature termination of specific types of mental operations. In the frequency domain, reduced synchronization was consistently observed in the theta band. Theta activity during wakefulness has been associated with working memory functions. Conclusions: The observation that a subset of brain states, and therefore assumingly a subset of networks show reduced synchronization of their active elements fits well with the hypothesis of a syndrome of functional disconnection that has previously been postulated to underlie the pathophysiology of schizophrenia. 


\section{S3.4}

\section{Treatment Effects on Resting State EEG in Schizophrenia}

M. Kikuchi' , T. Koenig ${ }^{2}$, Y. Wada ${ }^{3}$, M. Higashima ${ }^{1}$, Y. Koshino', W. Strik ${ }^{2}$, T. Dierks ${ }^{2}$

'Department of Psychiatry and Neurobiology, Graduate School of Medical Science, Kanazawa University, Kanazawa, Japan; ${ }^{2}$ Department of Psychiatric Neurophysiology, University Hospital of Psychiatry, Bern, Switzerland; ${ }^{3}$ Department of Neuropsychiatry, University of Fukui, Faculty of Medical Science, Fukui, Japan

Time domain analysis of electroencephalography (EEG) can identify subsecond periods of quasi-stable brain states. These socalled microstates assumingly correspond to basic units of cognition and emotion. On the other hand, Global Field Synchronization (GFS) is a frequency domain measure to estimate functional synchronization of brain processes on a global level for each EEG frequency band. The purpose of this study was to investigate changes of these EEG parameters after drug treatment in drug naïve schizophrenia. EEG analysis was performed in 14 drug-naive patients and was reevaluated 2-8 weeks (mean 4.3) after the initiation of drug administration. The present study extended findings of neuroleptic treatment effect on brain functions in schizophrenia by supplying the high time resolution necessary to study human information processing, or by supplying the high frequency resolution necessary to study synchronization of cortical networks, and imply that shortened duration of specific microstate classes seems a state marker especially in patients with later neuroleptic responsive, while lower theta GFS seems a state-related phenomenon and that higher gamma GFS is a trait like phenomenon.

\section{Session 4: Sleep EEG in Translational Research from Neuroscience to Clinical Psychiatry}

\section{S4.1 \\ SLEEP-EEG Characteristics of Antidepressants in the Mouse Model}

M. Kimura

Max Planck Institute of Psychiatry, Munich, Germany

Depressed patients frequently experience sleep disturbances, but more evident changes in their impaired sleep can be detected in their EEG traces such as shorten REM sleep latencies and lowered power densities in slow-wave activities. Meanwhile, effects of antidepressants are documented often to suppress REM sleep, but the effects on nonREM sleep are rather obscure. Recently we tested in normal C57BL/6J mice different classes of antidepres- sants, 2 tricyclic drugs: clomipramine or trimipramine, a selective noradrenaline reuptake inhibitor: riboxetine, and a selective serotonin reuptake inhibitor: escitalopram, and characterized whether they elicit significant modulation in specific EEG frequencies. Polygraphic data were processed by the LabVIEW-based acquisition program, in which an FFT algorithm served for the analysis of the particular EEG frequency contents. Clomipramine and reboxetine suppressed REM sleep but increased nonREM sleep. Within $15 \mathrm{~min}$ after injection, sharp increases in delta power and broad reduction of theta power were evident especially after the high dose. The magnitude of altered EEG was larger and the drug effects remained longer upon REM sleep than nonREM sleep. Trimipramine did not clearly affects sleep EEG at the low dose, but the high dose totally suppressed the whole power of all bands. Escitalopram only at the high dose reduced REM sleep but did not alter any nonREM-sleep parameters. The results suggest that common features in EEG changes can be detected during the treatment of different antidepressants. Although the suppression of REM sleep and theta power is a significant sign for monoaminergic drugs, a shifted peak of increased delta power could be additionally proposed as a potential marker evaluating central effects of the drugs.

\section{S4.2}

Sleep EEG and Antidepressant Treatment: Short-Term Outcome and Long-Term Course

\section{Hatzinger ${ }^{1}$, S. Brand, K. Baumann, U.M. Hemmeter ${ }^{2}$, M. Ising, E. Holsboer-Trachsler ${ }^{3}$}

${ }^{1}$ Hospital, Psychiatric Out-Patient Department, University Hospital, Basel, Switzerland; ${ }^{2}$ Cantonal Psychiatric Hospital, Wil/ St. Gallen, Switzerland; ${ }^{3}$ Psychiatric University Clinics Basel, Depression Research, Sleep Medicine and Neurophysiology, Basel, Switzerland

Introduction: In acute depression characteristic changes in EEG sleep measures are well documented findings. However, the course and the predictive value of these alterations for treatment outcome as well as for long-term course of depression still warrants clarification. Therefore, we examined whether (1) the previous clinical course of depression, (2) treatment response during a standard antidepressant therapy, and (3) the long-term outcome in follow-up are associated with abnormal EEG sleep parameters. Since the hypothalamic-pituitary-adrenocortical (HPA) system seems to play a crucial role in treatment outcome and course of depression, we evaluated HPA system function as well. Methods: 15 patients ( 4 men, 11 women; age 43-59) with depression were enrolled in the study. HPA system assessment using the combined DEX/CRH test and sleep EEG studies were conducted at baseline, after a 6 week antidepressant treatment period (trimipramine), and at follow-up i.e. after 2 to 10 years after the index episode. Results: 1. The previous clinical course as reflected by the number of episodes until baseline correlated significantly with EEG sleep measures i.e. sleep continuity values, slow wave sleep (SWS) and REM latency. 2. During treatment sleep continuity values improved and the correlation with the previous long-term course disappeared. 
However, the correlation with SWS persisted. The only sleep EEG marker at baseline predictive for treatment response was REM latency. 3. In the prospective long-term outcome SWS and REM density variables were related to the occurrence of recurrences in follow-up. These identified sleep EEG markers correlated closely with HPA system regulation. Conclusions: The previous and the prospective long-term course of depression are related to sleep EEG variables during the acute depressed state at the beginning and the end of treatment. Among the different values SWS and REM density measures seem to reflect predictive markers for the long-term course of depression. These markers are associated with HPA system regulation.

This work was supported by the Swiss NSF, 3200-052778.97

\section{S4.3 \\ Pharmacological Challenges During Sleep Deprivation in Depression}

\section{E. Holsboer-Trachsler', F. Muheim ', J. Beck' , M. Hatzinger², U.M.Hemmeter ${ }^{3}$}

${ }^{1}$ Psychiatric University Clinics Basel, Department of Depression Research, Sleep Medicine and Neurophysiology,Basel, Switzerland; ${ }^{2}$ Psychiatric Out-Patient Department, University Hospital, Basel, Switzerland; ${ }^{3}$ Cantonal Psychiatric Hospital, Wil/ St. Gallen, Switzerland

Sleep deprivation (SD) has an antidepressive, but temporary efficacy in $60 \%$ of depressed patients. Characteristic sleep EEG alterations of depression are improved after SD in the recovery night due to increased NonREM pressure. Naps and short microsleep (MS) episodes during SD reduce NonREM pressure in the recovery night and can prevent the antidepressant effect (Hemmeter et al. 1998). Based on these findings pharmacological manipulations to reduce MS during SD may intensify the accumulation of NONREM sleep pressure and augment antidepressant response. In a first study flumazenil, a GABA-A-benzodiazepine receptor antagonist, was applied in a double blind placebo-controlled design to depressed patients during partial SD (PSD). A substantial suppression of MS during PSD and an intensification of NonREM sleep in the recovery night could be achieved (Hemmeter et al. 2007). Modafinil, a new psychostimulant is able to reduce daytime sleepiness without direct GABA antagonistic effects. There is evidence that modafinil may exert its action via the hypocretin system, which is altered in major depression. To test the effect of modafinil on antidepressant response to PSD in depressed patients a placebo controlled, combined within / between subjects design has been conducted. For the evaluation of sleep EEG (before and after PSD) and MS episodes (at baseline and during SD), an ambulatory EEG recording system has been used to monitor for 60 hours the occurrence of MS episodes. Psychometric evaluation by Hamilton depression and visual analogue rating scales was performed before, during and after PSD. The different effects of flumazenil and modafinil will be discussed regarding sleep-wake regulation and antidepressant mechanism of SD.

\section{References}

Hemmeter U, Bischof R, Hatzinger M, Seifritz E, Holsboer-Trachsler E: Microsleep during Partial Sleep Deprivation in Depression. Biol Psychiatry 1998;43:829-839

Hemmeter U, Hatzinger M, Brand S, Holsboer-Trachsler E: Effect of flumazenil augmentation on microsleep and mood in depressed patients during partial sleep deprivation. J Psychiatric Res 2007;41:876-884

This work was supported by Swiss National Science Foundation and Cephalon $\mathrm{GmbH}$

\section{S4.4 \\ Effects of Peptides on Human Sleep EEG}

\section{A. Steiger, I.A. Antonijevic, M. Kluge, H. Murck, P. Schüssler}

Max Planck Institute of Psychiatry, Munich, Germany

A bidirectional interaction exists between sleep electroencephalogram (EEG) and nocturnal hormone secretion. Preclinical studies suggest that neuropeptides are common regulators of sleep EEG and sleep associated hormone secretion. In a series of studies we investigated the effect of various neuropeptides on sleep EEG and hormone secretion in normal control subjects and patients with depression. Peptides were injected in a pulsatile fashion intravenously. Sleep EEG and the secretion of cortisol and growth hormone $(\mathrm{GH})$ were investigated simultaneously. The key hormone of the somatotropic system, GH-releasing hormone (GHRH) increased Non-rapid-eye-movement (NonREM) sleep and GH and decreased cortisol in normal and depressed men, whereas the key hormone of the hypothalamo-pituitary-adrenocortical (HPA) system, corticotropin-releasing hormone $(\mathrm{CRH})$ exerted opposite effects. Sleep EEG of depressed patients normalized after a CRH antagonist. The effect of GHRH on sleep is influenced by gender, age and time of administration. GHRH prompted CRH like effects in women. In the elderly sleep promotion after GHRH was only weak. During the early morning, when endogenous HPA activity is high, sleep remained widely unchanged after GHRH. During the recovery night after sleep deprivation GHRH and CRH as well augmented the sleep promoting effect of sleep deprivation. In normal male subjects also ghrelin, galanin and neuropeptide $\mathrm{Y}$ promoted sleep, whereas somatostatin impaired sleep. Vasoactive intestinal polypeptide decelerated sleep cycles. Pituitary adenylate cyclase activating polypeptide (PACAP) appears to participate in homeostatic sleep regulation. Our data suggest that various peptides exert specific effects on human sleep EEG. A reciprocal interaction of GHRH and CRH appears to play a key role in sleep regulation, at least in male subjects. Changes in the GHRH/CRH ratio in favour of GHRH appear to contribute to the aberrances of sleep during depression (CRH overactivity) and ageing (reduced GHRH activity). The peptidergic regulation of spontaneous and recovery sleep appear to be different. 


\section{S4.5 \\ Effects of Choline Esterase Inhibitors on Sleep-EEG in Dementia}

U. Hemmeter ${ }^{1,5}$, A. Thum ${ }^{1}$, R. Rocamora ${ }^{1,3}$, M. Giesler ${ }^{1}$, A. Haag ${ }^{1,2}$, R. Dodel ${ }^{2}$, T. Penzel', J.C. Krieg ${ }^{1}$

${ }^{1}$ Cinic of Psychiatry and Psychotherapy, University of Marburg, Germany; ${ }^{2}$ Clinic of Neurology, University of Marburg, Germany; ${ }^{3}$ Hospital Psiquiatric Universitari Institut Pere Mata, Reus, Spain $;{ }^{4}$ Clinic of Internal Medicine, Dept. of Pneumology, University of Marburg, Germany; ${ }^{5}$ Center of Education and Research, Psychiatric Services Wil/St.Gallen,

Switzerland

Besides cognitive impairment, patients with dementia commonly present with a disturbance of sleep. A reduction of REMsleep is a characteristic finding in Alzheimer's dementia (DAT). Cholinsterase inhibitors (Che-I) the treatment of choice in mild/ moderate DAT are able to increase REM-sleep in volunteers and DAT. To date, there is only one sleep-EEG study in Alzheimer's disease concerning long term treatment, no data are available for fronotemporal dementia (FTD). In 22 patients with DAT and 8 with FTD who were free of psychotropic medication a polysomnography was performed. A subgroup of this sample 5 men, 2 women (age 68.1 \pm 8.0, MMSE 23.0 \pm 4.2 ) with DAT and 4 men, 2 women, age $(56.8 \pm 10.6$, MMSE $23.4 \pm 3.7)$ with FTD who were on monotherapy with a cholinesterase inhibitor (Che-I) could be re-examined after several months. In all patients a marked sleep continuity disruption was observed. Patients with DAT presented with a REM sleep reduction in comparison to FTD. Treatment with Che-I did not improve sleep continuity. REM-sleep increased in patients with DAT, but not in patients with FTD. The observed REM-sleep reduction in patients with DAT compared to FTD may suggest that cholinergic neurotransmission is more impaired in DAT than in FTD. However, it remains unclear whether the increase of REM-sleep after long-term treatment with Che-I in DAT may reflect a mere pharmacological effect or whether this increase may be related to the efficacy of anti-dementia treatment.

\section{Session 5: Relevance of Pharmaco-EEG: A Debate between Academia and Industry}

\author{
S5.1

\section{Open Discussion Paper: Absence-Status Epilepticus Caused by Cannabinoids?}

C.M. Van Rijn' ${ }^{1}$, R. Ngomba ${ }^{2,3}$, I. Midzyanovskaya ${ }^{4}$, S. Gaetani' ${ }^{3}$, G.D. Kuznetsova ${ }^{4}$

${ }^{1} \mathrm{NICl}$, Donders Institute for Brain and Cognition, Radboud University Nijmegen, The Netherlands, ${ }^{2}$ N.M. Neuromed, Pozzilli; Italy, ${ }^{3}$ Dept. of Pharmacology and Physiology, University of Rome, La Sapienza', Italy, ' Institute Higher Nervous Activity and Neurophysiology/RAS, Moscow, Russia

The audience will be asked to actively contribute to the discussion concerning the mechanism that underlies an observed absence-like status epilepticus in rats after a single dose of a cannabis agonist. Clarifying this mechanism may help to answer the intriguing, yet unsolved question what causes epileptic seizures to stop at all. Activation of pre-synaptic cannabinoid CB1 receptors inhibits neurotransmitter release. The neurophysiologic consequences of this activation thus depend on whether $\mathrm{CB} 1$ receptors are located on excitatory or inhibitory pre-synaptic terminals. Abnormalities in excitatory and/or inhibitory neurotransmission in the cortico-thalamo-cortical circuitry are thought to be one of the mechanisms underlying absence epilepsy. The role of the cannabinoid system in regulating non-convulsive seizure activity has not yet been investigated however. Here, we used the genetically epileptic WAG/Rij rat model, which shows spontaneous non-convulsive seizures, to examine whether pharmacological activation of CB1 receptors affects absence seizures. We recorded spontaneous spike-wave discharges (SWDs) in WAG/Rij rats injected with solvent or with the potent cannabinoid agonist WIN55,212. A single dose of WIN55,212 (3-12 mg/kg, i.p.) reduced the incidence of SWDs during the first 1-3 hours post-treatment in a dose-dependent way. After this initial reduction, the incidence of the SWDs returned to baseline level. However, the duration of the reappearing SWDs was markedly changed. Multiple very long SWDs, absence status-like periods, were observed: SWDs during up to 100 seconds while baseline SWDs are rarely longer than 17 seconds. These status-like periods were seen for at least 48 hours after administering the single dose. Possible mechanisms for this phenomenon will be postulated. The relation with GABA will be discussed, since enhancement of the endogenous GABA concentration by vigabatrin induces several long SWD periods as well. The possibility of trapped active metabolites will also be considered. 
S5.2

\section{Theta Band LFP Coherence Between Hippocampus And Prefrontal Cortex and Reorganization of Ensemble Cell Activity During Learning}

K. Benchenane, M. Khamassi, A. Peyrache, F.P. Battaglia, S.I. Wiener

Collège de France, Paris, France

Technological advances now permit simultaneous recordings of over 100 channels of neuronal and local field potential activity in several brain structures in the behaving rat. This permits direct observation of signal processing underlying cognitive processes such as learning, recall, and decision making. However, these recording technologies must be complemented by advances in analytical techniques for the ensemble recording data. Specific examples will be demonstrated of the recording technologies and novel analytical tools and their application to studies of the neural bases of learning in the hippocampo-prefrontal pathway.

\section{S5.3}

\section{Stress: A Neural Disconnection Syndrome - Can Electrophysiology Rescue Drug Discovery?}

\section{Spedding ${ }^{1}$, T.M. Jay², C. Sebban ${ }^{3}$}

${ }^{1}$ IdR Servier, Suresnes, France ; ${ }^{2}$ INSERM U796, Hopital Sainte Anne, Paris, France $;{ }^{3}$ Hopital Charles Foix, Ivry sur Seine, France

Virtually all the receptors coupled to G proteins (GPCRs) in the human genome have been published but there are an immense number of variables which can result in real functional differences between drugs, even with similar affinities, e.g. functional trafficking, the existence of receptor heterodimers, different accessory proteins. As it is difficult to predict the effects of drugs in a pathophysiological system in the brain from simple receptor affinities, a systems approach must be coupled to drug discovery to derive the brain areas involved in psychiatric disorders, and to model dysfunction in these systems using biomarkers (imaging, EEG, electrophysiology). Severe stressors change plasticity acutely (minutes) in key circuits leading to changes in connectivity and dendritic arborisation, and cellular and metabolic activation. Proof of principle studies have shown stress-mediated dysfunction in the key systems predicting anxiolytic, depression and schizophrenia : plasticity is reduced in hippocampus - medial prefrontal cortex (H-PFC, context) and increased in amygdala - orbitofrontal cortex (fear, emotive aspects), and periacqueductal grey (PAG). These areas are critical for cognition, emotion and susceptibility to psychiatric disorders (Agid et al., 2007) and the intracellular cascades involved are known. Acute stress inhibits hippocampal-PFC LTP in vivo in the rat and acute antidepressants, or clozapine (but not haloperidol) administration restores LTP. Effects on memory and emotive processing go in parallel with the model. These effects are only partly driven by glucorticoids. The findings were extended to include EEG in hippocampus and prefrontal cortex, evaluating theta rhythm coherence (and LTP) as a measure of plasticity between brain areas. Many drugs were evaluated in EEG in con- scious rats and clinical and preclinical data correlated. However, screening of metabolically stable brain-penetrant drugs in these systems results in drug- rather than receptor-specific pharmacology.

\section{Reference}

Agid $\mathrm{Y}$ et al., How can drug discovery for psychiatric disorders be improved? Nat Rev Drug Discov. 2007 6:189-201.

\section{Session 6: EEG-Based Personalized Medicine}

S6.1

EEG Phenotypes Predict Treatment Outcome to Stimulants in Children with ADHD

M. Arns' ${ }^{1}$ J. Gunkelman' ${ }^{1}$, M. Breteler', D. Spronk ${ }^{1}$

${ }^{1}$ Brainclinics Diagnostics B.V. Nijmegen, The Netherlands; ${ }^{2} \mathrm{Q}$-Pro Worldwide, Crockett, Calif., USA; ${ }^{3}$ Radboud University

Nijmegen / EEG Resource Institute, Nijmegen, The Netherlands

This study demonstrates that the EEG Phenotypes as described by Johnstone, Gunkelman \& Lunt are clearly identifiable EEG patterns with good inter-rater reliability. Furthermore, it was also demonstrated that these EEG phenotypes occurred in both ADHD subjects as well as healthy control subjects. The Frontal Slow, the Slow Alpha Peak Frequency and the Low Voltage EEG Phenotype seemed to discriminate ADHD subjects best from the control group, however not significantly. The Frontal Slow group responded to a stimulant with a clinically relevant decreased number of false negative errors on the CPT. The Frontal Slow and Slowed Alpha Peak Frequency phenotypes, have very different etiologies as evidenced by the treatment response to stimulants. In previous research the slowed alpha peak frequency has most likely erroneously shown up as a Frontal Theta sub-group. This implies that future research employing EEG measures in ADHD should avoid using traditional frequency bands, but clearly dissociate slowed alpha peak frequency from frontal theta by taking the individual alpha peak frequency into account. Furthermore, the divergence from normal of the frequency bands pertaining to the various phenotypes is greater in the clinical group than in the controls. Investigating EEG Phenotypes seems to be a promising new way to approach EEG data, explaining much of the variance in EEG's, and thereby potentially leading to more specific prospective treatment outcomes. 


\section{S6.2}

\section{EEG Susceptibility Markers in Personalized Medicine for Depression and ADHD}

\section{K.J. Barnett}

Brain Resource, Ultimo, NSW,Australia

This talk will discuss the opportunities and challenges for utilizing neuroscientific data in Personalized Medicine. The harsh reality is that treatments don't work for many people and they cause side effects in a significant percentage of patients. We will discuss Personalized Medicine and the diagnosis and treatment of brain disorders, with a focus on Depression and ADHD. The Brain Resource International Database (BRID) is a large standardized international database, with access to major data collection laboratories worldwide. A key differentiator of Brain Resource is "standardization" of all its methodologies. Each laboratory has identical set-ups and procedures to ensure comparability of data collected. This talk will present BRID data from a large cohort of depressed $(n=128)$, ADHD $(n=345)$ and matched controls. Neuropsychological data were collected using the standardized psychometric test battery while autonomic, and electrophysiological data were concurrently recorded. In both groups, electrophysiological changes differentiated clinical subjects from controls. We report the key EEG Markers that separated the clinical cohorts (e.g., raised Beta in depression and raised Theta in ADHD). Twenty eight depressed and fifty ADHD subjects underwent testing pre and post pharmacological treatment. The data show that EEG/ERP Markers can be linked with body-brain Markers to predict treatment response. BRID data captures temporal processing differences that lie on a continuum from milliseconds (e.g., startle responses) to seconds (e.g., skin conductance responses). We discuss susceptibility Markers in depression and ADHD in the context of our continuum model of emotion significance processing, which spans millisecond time scales at which automatic and arguably nonconscious processing occurs (e.g., the N1 startle response in depression) to seconds in which conscious processing occurs (e.g., the delayed P300 in ADHD). Our goal is to continue to validate treatment response Markers in psychiatric illness and prove their ecological validity.

\section{S6.3 \\ Referenced-EEG (rEEG) Guided Medication Selection for Psychotropics}

\section{D.A. Hoffman}

Neuro-Therapy Clinic, P.C. / CNS Response, Inc.,Greenwood Village, Colo., USA

Referenced-EEG is a database of medication correlations to brainwave patterns (biomarkers) that guide the practitioner in the selection of psychotropic drugs for the non-psychotic population. Current research for this evidenced based, personalized medicine track will be presented that will include use in general psychiatric disorders, eating disorders, dual diagnosis, ADHD and treatment resistant patients. This will be highlighted with case examples as well as how to use the rEEG report to augment clinical judgment. Future directions for the field will also be presented.

\section{S6.4 \\ Pre- and post-QEEG, ERP and Behavioural Effects of Left Frontal Magnetic Stimulation (rTMS) in Depression: Implications for Personalized Medicine}

D. Spronk ${ }^{1}$, M. Arns ${ }^{1}$, A. Bootsma ${ }^{1}$, R. Van Ruth ${ }^{1}$, P. Fitzgerald ${ }^{2}$

${ }^{1}$ Brainclinics Diagnostics B.V. / Brainclinics Treatment B.V. Nijmegen, The Netherlands; ${ }^{2}$ Alfred Psychiatry Research Centre, The Alfred and Monash University School of Psychology,

Psychiatry and Psychological Medicine, Melbourne, Australia

rTMS treatment for Depression has been under investigation in many controlled studies over the last 20 years. Most studies used a single stimulation protocol to treat all patients in the same way. Little is known about inter-individual differences, contraindications for treatment as well as the neurobiological action of rTMS in patients. We therefore developed a personalized stimulation protocol based on the QEEG and neuropsychological data and also investigated pre- and post treatment effects on QEEG and neuropsychology. rTMS treatment was applied in 8 subjects for a maximum of 20 sessions to the Left Dorsolateral Prefrontal Cortex. Prior to treatment clients were assessed on a full QEEG and neuropsychological evaluation. Clients were stimulated over the left DLPFC with $10 \mathrm{~Hz}$ rTMS. All subjects showed full remission within 20 sessions and there was a $65 \%$ reduction in Depressive symptomatology (BDI score) in 15 sessions. There was also a clear decrease in the Neuroticism scale of the NEO-FFI personality questionnaire. Pre- and post-QEEG measurements did not reveal treatment specific effects, but only an indirect right frontal increase in delta power. On the other hand, ERP measures did reveal treatment specific effects by showing an increased positivity in the post-treatment ERP's specifically left frontal. The P2 amplitude demonstrated a significant left frontal increase in amplitude, whereas for the negative $\mathrm{N} 1$ and $\mathrm{N} 2$ a significant decrease in amplitude was observed. The results of this pilot study demonstrate that rTMS can be a safe and efficacious treatment modality for depression. Furthermore, a specific left frontal increase in positivity for the ERP's were found (increase P2 and decreased N1 and N2 components) most likely related to the rTMS over the left DLPFC. Furthermore, there was no change in the alpha asymmetry lending support to the fact that frontal alpha asymmetry can be considered a trait marker for depression. The findings from this pilot study require future replication with larger sample sizes. The updated BDI results from the entire patient population to date will be presented as well. 


\section{Session 7: EEG Studies in the Ketamine/ NMDA Model of Cognitive Impairments and Schizophrenia}

\author{
S7.1 \\ The Effects of Ketamine and Haloperidol on Human \\ Selective Attention \\ B. Oranje ${ }^{1}$, C.C. Gispen-de Wied ${ }^{2}$, H.G.M. Westenberg ${ }^{2}$, C. Kemner ${ }^{2}$, \\ M.N. Verbaten ${ }^{3}$, R.S. Kahn ${ }^{2}$ \\ ${ }^{1}$ Center for Neuropsychiatric Schizophrenia Research, \\ University Psychiatric Center Glostrup, Glostrup University \\ Hospital, Denmark; ${ }^{2}$ Department of Psychiatry, University \\ Medical Center, Utrecht, The Netherlands; ${ }^{3}$ Department of \\ Psychopharmacology, Faculty of Pharmaceutical Sciences, \\ Rudolf Magnus Institute for Neurosciences, Utrecht University, \\ The Netherlands
}

Background: In a previous study of our laboratory ketamine (a non-competitive N-methyl-D-aspartate (NMDA) antagonist) was found to induce similar psychophysiological anomalies in healthy volunteers as are generally found in patients with schizophrenia, i.e. reduced processing negativity (PN) and reduced P300 amplitude. In the current study it was investigated whether the typical antipsychotic haloperidol (D2 antagonist) could counterbalance these ketamine induced anomalies. Methods: In a randomized, double-blind, placebo-controlled experiment 18 healthy male volunteers received placebo/placebo $(\mathrm{P} / \mathrm{P})$, placebo/ketamine $(0.3$ $\mathrm{mg} / \mathrm{kg}$, i.v. $)(\mathrm{P} / \mathrm{K})$ and haloperidol $(2 \mathrm{mg}) / \mathrm{ketamine}(0.3 \mathrm{mg} / \mathrm{kg}$, i.v.) $(\mathrm{H} / \mathrm{K})$ on three separate test days, after which they were tested in an PPI-, P50 suppression- and an auditory selective attention paradigm. Results: Haloperidol/ketamine $(\mathrm{H} / \mathrm{K})$ reduced task performance compared to $\mathrm{P} / \mathrm{P}$, while no differences were found between $\mathrm{P} / \mathrm{P}$ and $\mathrm{P} / \mathrm{K}$. Placebo/ketamine reduced $\mathrm{PN}$ and $\mathrm{P} 300$ amplitude, compared to $\mathrm{P} / \mathrm{P}$ (PN and P300) and $\mathrm{H} / \mathrm{K}(\mathrm{PN})$. No difference in $\mathrm{PN}$ was found between $\mathrm{H} / \mathrm{K}$ and $\mathrm{P} / \mathrm{P}$. No difference in P300 amplitude was found between $\mathrm{P} / \mathrm{K}$ and $\mathrm{H} / \mathrm{K}$. Conclusions: Since $H / K$ disrupted task performance while $P / K$ did not, this reduction seems solely attributable to the effect of haloperidol, most likely by reducing prefrontal dopaminergic activity. This in turn may indicate that the abnormal task performance as is generally found in schizophrenia results from reduced frontal dopaminergic activity. Haloperidol did counterbalance the ketamine induced disruption of PN, but not that of the P300 amplitude. This suggests that the P300 is modulated by glutamatergic activity. Consequently, the reduction in P300 amplitude of schizophrenia patients might be due to decreased glutamatergic activity. Both glutamatergic NMDA and dopaminergic D2 receptors seem to be involved in $\mathrm{PN}$.

\section{S7.2 \\ Effect of Clozapine and Ketanserin Pretreatment on S-Ketamine-Induced Symptoms and Brain Activation in Heathy Humans}

\begin{abstract}
F.X. Vollenweider ${ }^{1}$, M. Benz' ${ }^{1}$, V. Treyer ${ }^{2}$, G. Duncan² ${ }^{2}$ D. Hell ${ }^{1}$
${ }^{1}$ University Hospital of Psychiatry, Department of Clinical Research, Zurich, Switzerland; ${ }^{2}$ University Hospital Zurich, Division of Nuclear Medicine, Zurich, Switzerland; ${ }^{3}$ Department of Psychiatry, University of North Carolina, Chapel Hill, N.C.,USA
\end{abstract}

Objects: To localize the functional neuroanatomy underlying S-ketamine-induced psychotic symptoms and attentional deficits and to invstigate the putative role of 5-HT2A receptors in NMDAantagonist model of psychosis. Methods: S-ketamine-induced psychopathological symptoms (OAV and BPRS) and alterations in regional brain activity was assessed in healthy volunteers by means of $\left[\mathrm{H}_{2}{ }^{15} \mathrm{O}\right]$ PET. 20 male subjects were tested in a with-in subject design: 10 receiving once placebo, S-ketamine $(0.06 \mathrm{mg} / \mathrm{kg} /$ $\mathrm{min}$ ), clozapine (30 $\mathrm{mg} \mathrm{po}$ ), and S-ketamine plus clozapine; the other 10 receiving placebo, S-ketamine, ketanserin (50 mg po), and S-ketamine plus ketanserin. Results: S-ketamine $(0.06 \mathrm{mg} / \mathrm{kg} /$ min) administration in healthy subjects mimicked a number of positive symptoms compared to placebo $(n=20)$. Concomitantly with these symptoms, S-ketamine increased regional brain activity in an extended network including prefrontal cortex, anterior cingultate, G. pallidus, thalamus, left temporomedial and insular cortex as well as in subcentral and brainstem regions. Reduced activity was found in parietal and occipital cortices and cerebellum $(\mathrm{p}<0.00001)$. Pretreatment with Clozapine $(30 \mathrm{mg})$ moderately reduced S-ketamine induced positive symptoms and partially reversed the S-ketamine-induced alterations in the anterior cingulate, insula, and temporal regions (BA 21), and partially in the cerebelllum. Morover, clozapine reduced brain activity in left hippocampus and bilaterally in parahippocampal structures and increased the activity in the pons and orbitofrontal cortex. Pretreatment with ketanserin (50 mg) partially reversed S-ketamine-induced alterations the left insula, putamen (G. pall), left inferior frontal lobe, anterior cingulate, cerebellum and pons. Conclusion: The present findings support the view that a disruption of fronto-striato-thalamo-cerebellar pathways may underlay ketamine-induced psychotic symptom formation similarly to that seen in some patients with acute schizophrenia (Liddle et al. 2000, Lathi et al. 2006).

S7.3
NMDAR Antagonists Induce Persistent Abnormal
Gamma Oscillations: Behavioral, Network and
Cellular Features
D. Pinault, E. Tolmacheva, J. Gaudias, T. Zheng
INSERM U666, Faculté de Médecine, ULP, Strasbourg, France
Cognitive dysfunction, hallucination and delusion are typical
sorders that are diagnosed in schizophrenic patients and that
disorders that are diagnosed in schizophrenic patients and that 
can be induced in humans following the administration of a single non-anesthetic dose of ketamine, a non-competitive NMDAr antagonist. What is the impact of a similar single injection of ketamine in the EEG of rodents? And what is the direct effect of ketamine in cortical networks? In awaked adult rats, a single subcutaneous injection of ketamine $(<5 \mathrm{mg} / \mathrm{kg})$ induces persistent aberrant gamma frequency $(30-80 \mathrm{~Hz})$ oscillations (increased power and intrinsic frequency) in the frontoparietal cortex and a concomitant ataxic behaviour. EEG recordings performed in deeply anesthetized rats demonstrated that these pathophysiological gamma oscillations are not caused by abnormal motor activity. Neither are they dependent on conscious sensorimotor processing. Local application of ketamine produces a cortical focus of persistent aberrant gamma oscillations, which progressively spread to adjacent networks. Furthermore, ketamine significantly increases the synchrony of basal gamma oscillations between two highly and not between two weakly interconnected structures. Juxtacellular recordings combined with EEG recordings have revealed that aberrant gamma oscillations are associated with a significant increase in the firing rate in the majority of glutamatergic corticofugal and thalamocortical neurons. Our findings suggest that ketamine-induced persistent gamma hypersynchrony is an aberrant network noise that might set out of control the spatiotemporal patterning of inputs in cortical-related networks, which would impair top-down processing. A persistent decrease or annihilation of the signal/noise ratio of the cognition-related transient gamma synchrony might cause cognitive dysfunction, acute psychosis and exacerbate schizophrenia symptoms. Therefore, this persistent aberrant gamma noise may be a potential neurophysiological marker of psychoses.

This work was supported by INSERM and ULP.

\section{S7.4 \\ Effects of NMDA Receptor Antagonist Memantine on Mismatch Negativity}

M. Korostenskaja ${ }^{1,2,3,4}$, V.V. Nikulin ${ }^{1,5}$, D. Kicic ${ }^{1,6,7}$, A.V. Nikulina ${ }^{1}$, S. Kahkonen ${ }^{1,2}$

${ }^{1}$ MEG Laboratory, Division of Neurology, Cincinnati children's Hospital Medical Center, Cincinnati, Ohio, USA; ${ }^{2}$ BioMag Laboratory, HUSLAB, Helsinki University Central Hospital, Helsinki, Finland; ${ }^{3}$ Cognitive Brain Research Unit, Department of Psychology, University of Helsinki, Finland; ${ }^{4}$ Department of Electrophysiology, Republican Vilnius Psychiatric Hospital, Vilnius, Lithuania; ${ }^{5}$ Department of Clinical Neuroscience, Karolinska Institutet, Clinical Neurophysiology, Stockholm, Sweden; ' ${ }^{6}$ aboratory of Biomedical Engineering, Helsinki University of Technology, Finland; ${ }^{7}$ Helsinki Brain Research Center, University of Helsinki, Finland

Mismatch negativity (MMN) and its magnetic counterpart $(\mathrm{MMN} m)$ have been shown to be altered in patients with various psychiatric and neurological disorders, e.g. Alzheimer's disease and schizophrenia, indicating deficits in involuntary attention. $\mathrm{N}$-Methyl-d-aspartate (NMDA) receptor-mediated glutamate dysfunction is suggested to underlie these deficits. However, the role of NMDA receptors in involuntary attention is poorly understood. Memantine is an NMDA receptor antagonist that has been demonstrated to be effective in the treatment of patients with Alzheimer's disease. We aimed to investigate whether a single dose of memantine would affect MMN/MMNm in healthy subjects studied with simultaneous electroencephalography (EEG) and magnetoencephalography (MEG). Monaural left-ear auditory stimuli were presented in a passive oddball paradigm with infrequent deviant tones differing in frequency and duration. Neuronal activity was recorded in 13 healthy subjects after oral administration of $30 \mathrm{mg}$ of memantine or placebo in a randomized, double-blind, cross-over design. MMNm was analyzed using equivalent current dipoles.MMNwas evaluated from frontocentral electrodes. Memantine lowered subjects' arousal level as measured by visual analog scales, and enhanced the amplitude of MMN in EEG. No differences in MMN latency were observed in MEG or EEG. Memantine did not affect the location, strength, amplitude or latency of $\mathrm{MMN} m, \mathrm{P} 1 m$, and $\mathrm{N} 1 m$ components. No changes in amplitude or latency were observed for P1 and N1 peaks. These results indicate that memantine affects involuntary attention without otherwise changing auditory processing of the stimuli. As memantine-induced changes in MMN were detected only in EEG, we suggest that the effect is mostly related to the frontal cortex.

\section{Session 8: Complementary Techniques Applied to Drug Neurophysiological Research in Healthy Volunteers}

\section{S8.1 \\ EEG-Loreta and Spect: Troubles on Cross-Modality Correspondence}

M.J. Barbanoj ${ }^{1,2}$, S. Romero ${ }^{1,3}$, J. Riba ${ }^{1}$

${ }^{1}$ CIM-Sant Pau, IR. Servei de Farmacologia Clínica, HSCSP, Barcelona, Spain; ${ }^{2}$ Departament de Farmacologia i Terapèutica, UAB, Barcelona, Spain; ${ }^{3}$ Centre de Recerca en Enginyeria Biomèdica, Departament ESAll, UPC, Barcelona, Spain

The complementary strengths and weaknesses of functional brain imaging methods based on hemodynamic responses (high spatial, low temporal resolution) and on neurophysiologically techniques (low spatial, high temporal resolution) make their combined use a promising strategy to improve our understanding of brain functions. However, this strategy requires a better understanding of the relationship between hemodynamic/metabolic and neuroelectric measures of brain activity. Results from two studies (one using EEG-LORETA ${ }^{(1)}$ and the other cerebral blood flow as measured by $99 \mathrm{~m}$ Tc ECD SPECT ${ }^{(2)}$ ) in healthy volunteers were used as starting point to weigh up cross-modality correspondence issues. In this studies measurements were obtained after ayahuasca consumption [South American psychotropic plant tea containing compounds with monoamine oxidase inhibiting and $5-\mathrm{HT}_{2 \mathrm{~A}}$ 
agonist activities] in double-blind, cross-over, placebo-controlled designs. Several topics will be discussed, such as, neurovascular coupling, physical and physiological basis underlying the techniques, signal-to-noise ratios, extra-missing-displaced sources, definition and biological basis of intrinsic (not stimulus- or task evoked) brain activity during resting wakefulness, multidimensionality of EEG based on spectral frequency bands, simultaneous vs. sequential multi-modal recordings. In this context, it will be concluded that it is not possible to advance to what extent the current lack of generally applicable rules of thumb connecting measures of brain electrical activity with cerebral blood flow is due to: (i) a genuine complexity between both measures, (ii) methodological shortcomings or (iii) insufficient theoretical development.

\section{References}

(1) Riba J et al. Neuropsychobiology 2004; 50: 89-101.

(2) Riba J et al. Psychopharmacology 2006; 186: 93-98.

The research team from CIM-Sant Pau is member of CIBERSAM (supported by Spanish Ministry of Health, Instituto de Salud Carlos III)

\section{S8.2 \\ The Estimation of Cortical Connectivity from High- Resolution EEG Measurements}

\section{F. Babiloni \\ University of Rome "Sapienza”, Dipartimento Fisiologia e Farmacologia, Roma, Italy}

Different functional connectivity methods for the estimation of cortical connectivity from neuroelectric and hemodynamic measurements have been applied on common data set in order to highlight similarities and differences in the obtained results. Such methods include Directed Transfer Function (DTF), Partial Directed Coherence (PDC) and direct Directed Transfer Function (dDTF). Comparisons will be made between these estimators in order to understand if, for a same dataset, functional connectivity estimators could return the same cortical connectivity patterns. In addition, connectivity patterns estimated with the use of only the neuroelectric information or the information from the multimodal integration of neuroelectric and hemodynamic data were compared. Results suggests that the estimation of the cortical connectivity patterns performed with the employed methods (DTF, PDC, dDTF) returned essentially the same information. Light differences in cortical connectivity between the patterns estimated with the use of multimodal integration were noted when compared to those estimated by using only the neuroelectric data.

\section{S8.3 \\ Complementary Techniques Applied to Drug- Neurophysiological Research: Event Related Potentials and Funcional Magnetic Resonance Imaging}

J. Riba

Centre d'Investigació del Medicament (CIM-Sant Pau), Institut de Recerca de l'Hospital de Santa Creu i Sant Pau, Departament de Farmacologia i Terapèutica, Universitat Autonòma de Barcelona, Spain

The brain networks and neurotransmitter systems involved in reward and punishment in motivational processing have been the object of increasing interest in recent years. Event-related brain potential studies have consistently demonstrated the appearance of frontocentral negativities when event outcomes are worse than expected. These frontocentral waves have typically been shown after performance errors in reaction time experiments, and also following monetary losses in gambling tasks. Whereas these electrophysiological correlates of negative outcomes can be reliably recorded and studied, no consistent wave has been related with reward processing. Functional magnetic resonance imaging (fMRI) has on the other hand found extensive brain areas to be responsive to monetary gains and positive feedback. Blood oxigenation level dependent response (BOLD) increases have been observed in an extensive network including the basal ganglia, orbitofrontal cortex, brainstem and anterior cingulate cortex when event outcomes are better than expected. These brain activations, however, do not seem to translate into recordable electrophysiological signals on the scalp surface. Analogously, monetary losses in gambling tasks do not lead to measureable BOLD changes in the frontomedial cortex, where the frontocentral negativities are thought to be generated. Two drug studies involving respectively electrophysiological recordings and fMRI in the context of motivational processing are presented in order to highlight the benefits of using complementary techniques when studying brain processes in humans.

The author is a member of CIBERSAM (supported by the Spanish Ministry of Health, Instituto de Salud Carlos III).

S8.4

Complementary Techniques Applied to DrugNeurophysiological Research: Population Pharmacokinetic/Pharmacodynamic Modeling

M. Valle, E. Grasa, M.J. Barbanoj

Centre d'Investigació del Medicament, Institut de Recerca de I'Hospital de la Santa Creu i Sant Pau,Departament de Farmacologia y Terapèutica, Universitat Autònoma de Barcelona, Spain

Pharmacokinetic/pharmacodynamic (PK/PD) modeling is the derivation of mathematical equations intended to describe 
observed data to elucidate the "in vivo" properties of drugs or the systems they interact with. The properties are described by means of parameters in such a manner that they allow predicting the effect in different situations. These may include the administration of the same drug under different conditions such as dosage design and whether the subject is healthy or ill. Thus, analyzing the time course of drug response using $\mathrm{PK} / \mathrm{PD}$ relationships represents nowadays one of the most efficient methods to deal with pharmacological effects. PK/PD modeling has been largely employed to describe data obtained from EEG and obtain "in vivo" pharmacological information of different drugs acting on the Central Nervous System (CNS) in both animals and humans. A selection of some basic aspects that can be covered using pharmacoEEG and PK/PD modeling from "in vivo" studies performed in animals and humans in pharmacological research will be introduced mostly based on studies performed with benzodiazepines and anesthetic drugs. A special case focus on the applicability to discriminate between placebo and drug effect will be presented in more detail.

The authors are members of CIBERSAM (supported by the Spanish Ministry of Health, Instituto de Salud Carlos III). M.Valle is supported by FIS through contract CP04/00121 from the Spanish Health Department in collaboration with Institut de Recerca de l'HSCSP.

\section{Posters}

\section{P1 \\ Effect of Subanesthetic Dose of Ketamine on The Brain Activity in Model of Schizophrenia li: A Pharmaco-Magnetic Resonance Imaging Study}

\section{J. Horacek, M. Brunovsky, B. Tislerova, T. Novak, F. Spaniel, J. Tintera, M. Dezortova}

Prague Psychiatric Center, Prague, Czech Republic; ZRIR, Institute for Clinical and Experimental Medicine, Prague, Czech Republic

Rationale: The glutamatergic hypothesis postulates that the $\mathrm{N}$-methyl-D-aspartate (NMDA) receptor hypofunction results in the schizophrenia symptoms. With respect to this hypothesis ketamine, the non-completive antagonist of NMDA is used to model schizophrenia in animals and in humans. The aim of our study was to detect the changes in brain activity followed subanesthetic dose of ketamine. Methods: In a double-blind, placebo-controlled pharmaco-magnetic resonance imaging study the effect of intravenous ketamine on blood oxygenation level-dependent (BOLD) signal was measured in the group of 11 healthy volunteers Ketamine was applied i.v. in the dose of $0.27 \mathrm{mg} / \mathrm{kg}$ within first $10 \mathrm{~min}$., followed by a infusion of $0.27 \mathrm{mg} / \mathrm{kg}$ for $20 \mathrm{~min}$ The imaging began $5 \mathrm{~min}$ utes before infusion and continued for a further 30 minutes (scanner: 3T Siemens Trio, ISI: $3 \mathrm{~s}$, isovoxel: $2 \mathrm{~mm}$, smoothing: $10 \mathrm{~mm}$ ). For the SPM5 analyses we used the "pseudoblock“design: 5 min- utes intervals before infusion were compared with $5 \mathrm{~min}$. intervals throughout the infusion of ketamine or placebo. In the 2nd level analysis, the contrasts form previous steps were compared with placebo (paired t-test, $\mathrm{p} \leq .01$ with FDR correction). Results: Ketamine induced increase of BOLD in a large cluster consisting of 2524 voxels in the right temporal lobe (superior temporal gyrus and insula) and in lesser extend also in the left temporal cortex. Conclusions: We confirmed that the NMDA antagonism increases activity in temporal and cingulate regions. Our results confirmed the role of cingulate and temporal regions in ketamine psychotogenic effect. The alteration in temporo-cingulate network would be responsible for the deficit of information processing in schizophrenia and ketamine model of this psychosis as well.

This work was supported by the grant No.NR-8792 (IGA MZ CR).

P2

\section{Prediction of Response to Cholinesterase Inhibitor Treatment in Alzheimer's Disease by Means of Sloreta}

\author{
M. Brunovsky, V. Krajca, A. Bartos, F. Diblikova, O. Vysata
}

Prague Psychiatric Center, Prague, Czech Republic; Dept. of Neurology, Faculty Hospital Bulovka, Prague, Czech Republic

Rationale: Despite the evidence-based effectiveness of cholinesterase inhibitors (ChEI) in the management of Alzheimer's disease (AD), a significant proportion of $\mathrm{AD}$ patients fail to response to this treatment. By means of standardized low-resolution brain electromagnetic tomography (sLORETA), we tried to identify a subpopulation of $\mathrm{AD}$ patients who can benefit from ChEI treatment maintenance. Methods: Resting EEG was recorded in 48 mild-tomoderate $\mathrm{AD}$ patients (mean age 78 years; $29 \mathrm{~F}$ and $19 \mathrm{M}$; MMSE 15-24) before and after 6 months on ChEI treatment. Based on the change of MMSE score after 2 years follow-up, 27 patients were classified as non-responders (decrease of MMSE $>2$ points) and 21 patients as responders. The localization of the differences in source distribution between both groups (at baseline) and within groups (baseline vs. 6 months) was assessed by voxel-by-voxel t-tests of sLORETA images of the log-transformed current density power in seven frequency bands. Results: At baseline, responders had significantly lower current density in delta and alpha frequency band, which sLORETA localized in frontal (BA 6,8,9,32,46) and temporo-parieto-occipital areas (BA 7,17,22,37,39,40). After 6 months of ChEI treatment responder group showed significant increase of beta current density sources mainly in the frontotemporal (BA $10,21,22,40$ ) and parahippocampal areas (BA 27,30,35,36), whereas non-responders showed decrease of alpha- 2 and beta- 1 sources predominantly in the frontal regions (BA 6,9,32). Conclusion: Our results suggest that there is a subpopulation of $\mathrm{AD}$ patients (probably with more pronounced central cholinergic deficiency syndrome) with better response to ChEI treatment, which can be identified by means of sLORETA.

The study was supported by grant IGA MZCR 1A8600. 


\section{P3 \\ The 5-HT1A/2A Agonist Psilocybin Disrupts Modal Object Completion Associated with Visual Hallucinations}

\section{Kometer, F.X. Vollenweider \\ University Hospital of Psychiatry,Clinical Research \& Heffter Research Center, Zurich, Switzerland}

Background: The 5-HT1A/2A agonist psilocybin is known for its ability to induce a psychotic state including visual hallucinations that resembles some of the signs of acute schizophrenia. In schizophrenia reduced 5-HT2A receptor density and disturbances in visual processing such as modal object completion associated with decrements in the P1 and/or N170 component have been reported. Methods: To further investigate the role of 5-HT1A/2A receptors in visual processing and hallucinations the effect of psilocybin $(115 \mu \mathrm{g} /$ and $215 \mu \mathrm{g} / \mathrm{kg}$ vs placebo) on modal object completion has been assessed using the Kanizsa figures and highdensity electrical mapping and source-analysis (LORETA) in normal volunteers $(n=17)$. Results: We found that psilocybin dosedependently impaired modal object completion associated with a preferential reduction of the N170 amplitude to the Kanizsa relative to Non-Kanizsa stimuli in occipital cortex (LOC). This finding was supported by an additional behavioural visual detection task. Moreover, the overall reduction of the N170 amplitude in the Kanizsa and the non-Kanizsa condition correlated with the degree of psilocybin-induced visual hallucinations. Conclusion: These results suggest that a disruption of the serotonergic neurotransmission, particularly at the level of the $5-\mathrm{HT} 1 \mathrm{~A} / 2 \mathrm{~A}$ receptor, leads to visual hallucinations and aberrant modal object completion by modulating the N170 component. Given that similar disruptions were found in schizophrenic patients the present results suggest that interferences at the level the 5-HT1A/2A receptor may underlay the aberrant object completion found in schizophrenia.

\section{P4 \\ Quantitative EEG in Glutamatergic and Dopaminergic Models of Psychosis - Animal Study}

T. Palenicek, M. Fujakova, M. Kutova, M. Brunovsky, J. Horacek, P. Sos, B. Tislerova, V. Bubenikova-Valesova

Prague Psychiatric Center, Prague, Czech Republic

Rationale: EEG abnormalities in schizophrenia predominantly include increased power in delta and theta frequency bands in frontal regions, a decrease in alpha and increase in beta power and changes in EEG coherences. To date there have only been a few studies describing qEEG changes in animal models of psychosis. We have focused on qEEG changes in glutamatergic (ketamine) and dopaminergic (amphetamine) models. Methods: Male Wistar rats were stereotactically implanted with 14 silver electrodes located in frontal, parietal and temporal cortical areas. EEG was recorded 7 days after surgery in freely moving animals. After the first baseline record of 10 min the rats were administered with either ketamine
$30 \mathrm{mg} / \mathrm{kg}$ i.p. or amphetamine $4 \mathrm{mg} / \mathrm{kg}$ s.c. and the signal was recorded for another $30 \mathrm{~min}$ with a BrainScope digital amplifier system. Artifact-free epochs of $30 \mathrm{~s}$ were selected from the baseline record and from two time intervals after administration of the drug. EEG power spectra and coherences were calculated and analyzed using Neuroguide software v. 2.4.6. The data from both time intervals after drug application were compared with their corresponding baseline values by means of paired t-tests. Results: Ketamine led to a massive cortical excitation demonstrated by an overall increase in absolute power in all cortical regions. Similarly, ketamine increased almost all coherences throughout the whole spectrum. Amphetamine increased power in theta, alpha and gamma frequency bands while a decrease was observed in theta and beta bands. Coherences were increased mainly in theta and alpha bands, while a decrease in coherences was observed in delta, beta and gamma bands.

This work is supported by projects IGA MHCR NR-8792-3, NR-8785-3, CNS MSMT 1M0517 and MZ0PCP2005

\section{P5 \\ Classification of Psychotropic Drugs: Validation of Neurophysiological Markers Projected in a High Dimensional Space}

M. Tohmé ${ }^{1,2}$, R. Lengellé ${ }^{2}$ P.H. Boeijinga

${ }^{1}$ FORENAP, Rouffach, France ; ${ }^{2}$ Institut Charles Delaunay-LM2S (FRE CNRS 2848), Université de technologie de Troyes, Troyes cedex, France

In this paper, we try to classify psychotropic drugs according to their efficacy. The difficulties of the problem reside essentially in the fact that traditional methods do not apply when observations are in a high dimensional space and, at the same time, the number of independent observations is small, which is a common case in biomedical research. In this case, usual methods fail to give a solution. In our application, effects of drugs on ERP signals, the number of variables considered is indeed higher than the number of volunteers. For instance, to evaluate an effect, at least 2 repeated measures are necessary, one before and one after drug intake (but more often 5 to 10 are used). We propose an alternative solution to usual group comparison tests by using a pattern recognition approach. In this approach we attempt to classify the observations within two groups. Consequently, the simplest classical approach, namely group mean testing is replaced by a hypothesis test on the classification error probability. As usual, the statistic that will be used to make the decision is an estimate of this error probability. We have evaluated different training algorithms (which induce different statistical properties of the chosen error probability estimate) in order to rank their stability. We also present experimental results relating stability and power functions of the tests based on the different training algorithms considered. The "best" analytical and simulation-based couple (error estimate, detector) that allows us to get the highest power function will be reviewed. Empirically, a realistic measure of the efficacy of sedative drugs on P300 will be dealed with as well. The impact on decision on drug efficacy and extension to multiclass will be discussed. 
P6

P300 Differences in Averaged Event Related Potentials and Event Related Oscillations: Ketamine, Apomorphine and the Model for an Endophenotype for Schizophrenia

P.H. Boeijinga, L. Soufflet, R. Luthringer

FORENAP, Rouffach, France

Schizophrenic patients have left hemisphere reductions in P300 amplitude, and delayed peak latencies. Meta-analysis has shown that P300 potential amplitude in an auditory paradigm is marginally attenuated in first degree relatives of Schizophrenic patients in e.g. siblings (e.g. Turetsky et al. 2000). We investigated in healthy volunteers whether Event Related Oscillations are more appropriate to characterize deficits in information processing than the amplitude measures. In relation to the NMDA hypothesis, research on ketamine effects has been carried out and will be presented. They will be compared to the effects of apomorphine and preliminary data on the interaction with neuroleptics. Moreover evaluations of the biomarker obtained in parents of patients will be shared with the audience. We hope so to develop deepened thoughts on the P300 endophenotype.

\section{Reference}

Turetsky BI, Cannon TD, Gur RE (2000) P300 subcomponent abnormalities in Schizophrenia : III. Deficits in unaffected siblings of schizophrenic probands. Biol Psychiatry 47; 380-390

\section{P7}

Evaluation of The Cognitive Function for Amyotrophic Lateral Sclerosis with Event-Related Potentials

T. Ogawa, H. Tanaka, R. Takashima, Y. Hoshino, Y. Watanabe, K. Hirata

Department of Neurology, Dokkyo Medical University, Mibu, Tochigi, Japan

Objectives: The purpose of this study was to determine the cognitive impairment profile of ALS using neuropsychological tests and event-related potentials (ERP). Methods: We recruited 19 patients with sporadic ALS (8 with spinal-onset ALS and 11 with bulbar-onset ALS) and 19 age-, sex- and education-matched normal subjects. Neuropsychological signature was assessed by Mini-Mental State Examination (MMSE), Hasegawa Dementia Scale-Revised (HDS-R), Wisconsin Card Sorting Test (WCST), Wechsler Adult Intelligence Scale-revised (WAIS-R), Stroop Test, and Trail Making Test (TMT). The results of ERP and neuropsychological tests were correlated with pulmonary function and clinical acuity. For ERP, the auditory "oddball" counting paradigm was presented during EEG recording. Global field power (GFP) was computed from all channels, and GFP peak amplitude and latency were determined. Results: The scores of MMSE, HDS-R, and WAIS-R indicated no obvious dementia in the cohort. No significant impairment was detected in other neuropsychological tests. In ERP study, N1/N2/P3 latencies were significantly prolonged in ALS than the control. Vital capacity correlated with P3 amplitude, and the relative bulbar functional rating scale correlated significantly with P3 latency. Conclusions: Our findings confirmed the presence of cognitive dysfunction in patients with sporadic ALS, especially those with bulbar-onset ALS, although pulmonary impairment seems to play part in impaired cognition regardless of ALS subtype.

P8

\section{Cortical Blood Flow Change in Migraine - A Study Using Near Infrared Spectroscopy}

Y. Watanabe, H. Tanaka, R. Takashima, S. Aiba, H. Iwanami, K. Kimoto, T. Ogawa, T. Komagamine, R. Okabe, K. Hirata

Department of Neurology, Dokkyo Medical University, Mibu, Tochigi, Japan

Migraine is representative of primary headache, and it is characterized by pulsating, paroxysmal, and sensitive to light and sound during attack. However, the pathopysiology is still unknown. The aim of our study is to evaluate cortical blood flow change in the patients with migraine during attack and before and after administration of sumatriptan. We selected two patients of migraine and one patient of tension type headache diagnosed by ICHD-2. In addition, one normal control was submitted. Data acquisition is how to measure the data using near infrared spectroscopy (NIRS) topography. We recorded multi-channel NIRS with ETG100 (Hitachi Medical Corporation, Japan) during headache attacks. We attached $3 \times 4$ probes for each hemisphere covered over the bilateral temporal and parietal lobes. The oxyHb data was sampled with $10 \mathrm{~Hz}$ (100 msec intervals). We used power spectrum analysis with FFT and autocorrelation to analysis the data. We evaluated standard deviation the oxyHb data before and after administration of sumatriptan. Spectral peak at $1.2 \mathrm{~Hz}$ was enhanced in right compared to left, which is agreed with migraine locus of this patient. Autocorrelation recognized peaks every $0.8 \mathrm{sec}$, which accepted enhancement of power in the right compared to the left. The difference appeared in SD value score between migraine and tension type headache. SD value is decreased by sumatriptan administration to migraine patient. SD value of control subject did not change by placebo. We conclude that NIRS might be useful and reliable method to detect fluctuation of blood flow during migraine attack. NIRS might be a KEY method for clarifying the pathopyhsiology of migraine. 


\section{P9}

\section{Cerebral Hypersensitivity of Migraine Patients Assessed by Photic Driving Responses in EGG}

\author{
R. Takashima, H. Tanaka, Y. Hoshino, T. Ogawa, Y. Watanabe, \\ K. Kimoto, K. Hirata \\ Department of Neurology, Dokkyo Medical University, Tochigi, \\ Japan
}

Photic supersensitivity is an aggravation factor of migraine attack and included in the diagnostic criteria. This study is aimed to investigate the mechanism in the brain electric signature of migraineurs, with the photic driving responses in the electroencephalogram (EEG). We studied 25 patients (male/female $=6 / 19$, age 15-58, migraine with aura/migraine without aura $=12$ / 13) who had a checkup at outpatient and diagnosed as migraine with the diagnostic criteria (ICHD-2). We recorded spontaneous eyes-closed resting EEGs from 20 electrodes on the scalp. Three artifact-free EEG epochs consisting of $2.56 \mathrm{~ms}$ during photic stimulation were selected. Stroboscope flashes consisting of 3-5-8$10-13-15-18-20 \mathrm{~Hz}$ were used. 18 in 25 patients had photic driving responses, and 13 among them had spread from occipital to frontal lobe. Standardized Low Resolution Electromagnetic Tomography (sLORETA) was used to compute the three-dimensional intracerebral distribution of electric activity for each driving response. sLORETA revealed significant activities related to driving response at not only occipital lobe but also frontal lobe. We conclude that higher rate of photic driving response in migraineurs is thought to reflect cerebral hypersensitibity, and it might concern to anterior cingulate and prefrontal cortex for sensibilization of limbic system.

\section{P10 \\ Analysis of Brain DC Potentials Improves the Understanding of Mechanisms of Action of Drugs in Patients with Vascular Encephalopathy}

\section{V.F. Fokin, N.V. Ponomareva, E.I. Ivashtenko, M.V. Krotenkova, R.N. Konovalov}

Research Center of Neurology of Russian Academy of Medical Science, Moscow, Russia

The brain DC-potential of $\mathrm{mV}$ range is mainly generated on the border of blood brain barrier (BBB) and can be registered from the surface of the head. The amplitude of DC potentials depends on the difference of concentrations of hydrogen ions on both sides of the $\mathrm{BBB}$, which depend on both intake of glucose and predominant type of glucose metabolism (aerobic or anaerobic). We studied 64 patients with vascular encephalopathy (VE) by using brain DC potentials, computed tomography perfusion and transcranial Doppler sonography. The examination were performed before and after one month administration of vinpotropil, which has nootrop properties and improves the cerebral glucose metabolism. Positive correlations were observed between regional cerebral blood flow, cerebral blood volume and amplitude of DC potentials, as well as between DC potentials and blood flow velocity in the main brain arteries. The amplitude of DC potentials in the patients with VE were elevated compared with the normal controls. After vinpotropil therapy together with a marked clinical improvement the normalization of DC potentials was observed. After treatment the blood flow velocity in the main brain arteries did not change significantly. It is possible that the decrease of brain DC potentials after the treatment is due to the reduction of cerebral acidosis connected with increase of aerobic metabolism of glucose.

\section{P11}

\section{Cerebral Hyperexcitability, Associated with Genetic Risk for Alzheimer's Disease}

\author{
N.V.Ponomareva1 , T.V. Boikova², G.I. Korovaiceva², E.I. Rogaev 2,3 \\ ${ }^{1}$ Research Center of Neurology RAMS, Moscow, Russia, \\ ${ }^{2}$ Research Centre for Mental Health RAMS, Moscow, Russia; \\ ${ }^{3}$ University of Massachusetts Medical School, Worcester, Mass., \\ USA
}

To search early brain alteration related to genetic susceptibility to Alzheimer's disease (AD) we studied quantitative EEG and apolipoprotein $\mathrm{E}$ (ApoE) genotype in 50 non-demented $\mathrm{AD}$ patients' (ADP) relatives and in 80 age-matched normal controls. In the persons with genetic risk for $\mathrm{AD}$ the examination was performed twice - before and after one-month administration of picamilon, which combines nootrop and tranquillisation properties. In the $\mathrm{ADP}$ relatives and in normal controls the presence of ApoE $\varepsilon 4$ allele was associated with excessive reactivity to hyperventilation, characterized by the manifestation of synchronous high-voltage delta-, theta-activity and sharp-waves. The significant differences in changes of delta, theta, alpha and beta powers between ADP relatives $\varepsilon 4$ allele noncarriers and healthy subjects were also detected suggesting the potential effect of other genetic factors. Nonetheless, in the ADP relatives carrying $\varepsilon 4$ allele the differences in delta and alpha powers were more pronounced. The EEG signs of hyperexcitability were more frequently observed in the relative of patients with early-onset, than late onset $\mathrm{AD}$. After one-month administration of picamilon the EEG alterations were reduced in the persons with genetic risk for $\mathrm{AD}$. The results suggest the involvement of aberrant neuronal overexcitation and excitotoxic mechanism in the neuronal injury in the persons genetically predisposed to AD.

This work was supported by RFBR. 


\section{P12 \\ Prefrontal Theta QEEG Cordance Value in Patients with Resistant Depressive Disorder - One Year Follow Up}

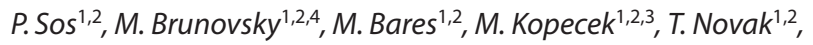
B. Tislerova ${ }^{1,2}$, V. Kraca ${ }^{4}$

${ }^{1}$ Prague Psychiatric Centre, Prague, Czech Republic; ${ }^{2}$ Department of Psychiatry and Medical Psychology, 3rd Faculty of Medicine, Charles University, Prague, Czech Republic;

${ }^{3}$ Center of Excellence for Research and Treatment Bipolar Disorder, Department of Psychiatry, University of North Carolina at Chapel Hill, N.C., USA; ${ }^{4}$ Department of Neurology, Faculty Hospital Na Bulovce, Prague, Czech Republic

Introduction: Our previous study of unipolar depression patients has shown that decrease of prefrontal theta QEEG cordance value after first week of venlafaxine treatment can predict clinical response [1]. In the prefrontal electrodes specifically, theta surface EEG activity has been associated with activity in underlying anterior cingulate cortex as measured by MEG and PET [2;3]. Previous research has linked pretreatment activity in the anterior cingulate cortex with clinical response to antidepressant medication $[4 ; 5]$. We have now examined whether change of prefrontal QEEG cordance value response to the clinical change after 1 year follow up in resistant depression patients. Methods: We analyzed 17 inpatients who endured single follow up after 1 year. EEG data were compared with one at the baseline and after 1 week of treatment. QEEG cordance was computed at three frontal electrodes in theta frequency band. Depressive symptoms and clinical status were assessed using MADRS, BDI and CGI. Results: Seven of 8 patients in remission (MADRS $\leq 13)$ and only 2 of 9 non-remitters had increased prefrontal QEEG cordance value after one year of depression treatment $(p=0.01)$. Conclusion: The increase of prefrontal QEEG theta cordance value after one year of treatment might be helpful in the prediction of maintenance of remission in depression patients.

\section{References}

[1] M. Bares, et al. Eur Psych, vol. 23, no. 5, p. 350-355, 2008.

[2] H. Asada, et al., Neurosc Lett, vol. 274, no. 1, pp. 29-32, 1999.

[3] A. M. Hunter, Psych Clin of North Am, vol. 30, no. 1, pp. 105-124, 2007.

[4] H. S. Mayberg, et al., Neuroreport, vol. 8, no. 4, pp. 1057-1061, 1997.

[5] D. Pizzagalli, et al., ICS, vol. 1232, pp. 711-717, 2002.

The study was supported by MZCR MZ0PCP2005

\section{P13}

\section{Effects of Atorvastatin and Pravastatin on the EEG}

\section{N.A. Wright, C. Turner, B.M. Stone}

Human Protection and Performance Enhancement, QinetiQ, Farnborough, Hampshire, UK

High plasma cholesterol is a major risk factor in heart disease, and thus lipid-lowering drugs such as statins can potentially reduce the incidence of myocardial infarction. Statins are frequently taken by individuals engaged in skilled activity, and so impaired alertness or mental performance is to be avoided. Aim:To establish whether the statins atorvastatin and pravastatin impair performance or alertness. Methods: Effects of pravastatin (20,40mg) and atorvastatin $(40,80 \mathrm{mg})$ were investigated using EEG, performance tests and subjective alertness from 0.5 to $7.75 \mathrm{~h}$ after ingestion in 9 healthy male volunteers. The study was placebo-controlled and double-blind, with promethazine $(20 \mathrm{mg})$ as active control. EEG was recorded from Fp1, F3, F4, P3, P4, O1, O2 during a digit memory recall task and an eyes-closed rest condition. Power in delta $(.5-3 \mathrm{~Hz})$, theta $(3.5-7 \mathrm{~Hz})$, alpha1 $(7.5-10 \mathrm{~Hz})$, alpha2 $(10.5-13 \mathrm{~Hz})$, beta1 $(13.5-21 \mathrm{~Hz})$, beta2 $(21.5-30 \mathrm{~Hz})$, gamma $(30.5-100 \mathrm{~Hz})$ bands was calculated from spectral estimates. Memory performance, attention, DSST and reaction time were tested, and alertness assessed subjectively. Results: Pravastatin (20, $40 \mathrm{mg})$ reduced beta and gamma activity in Fpl and F3 at 3.75-5h, suggesting adverse effects on mental activity. In the eyes-closed condition, atorvastatin ( $80 \mathrm{mg}$ ) increased theta in P3 and P4 at 0.5 and 3.75-6.5h, suggesting sleepiness. Promethazine reduced gamma during the memory task; in the eyes-closed condition, delta and theta increased and alpha, beta and gamma reduced. Pravastatin impaired memory recall, and with both drugs subjects reported tiredness. Conclusion: The findings suggest that statins should be used with caution at least initially by individuals engaged in skilled activity where maintaining performance and alertness is critical.

\section{P14 \\ The Effects of the Novel Anxiolytic Drug ABIO-08/01 on Spontaneous and Event-Related Brain Potentials}

\author{
P. Anderer ${ }^{1}$, G.M. Saletu-Zyhlarz' , M. Wolzt ${ }^{2}$, H.V. Semlitsch ${ }^{1}$, \\ A. Assandri ${ }^{3}$, P. Nessi ${ }^{3}$, F. Nannipieri ${ }^{4}$, S. Rosini ${ }^{4}$, B. Saletu ${ }^{1}$ \\ ${ }^{1}$ Department of Psychiatry and Psychotherapy and \\ ${ }^{2}$ Department of Clinical Pharmacology, Medical University of \\ Vienna, Vienna, Austria; ${ }^{3}$ Cross S.A. - Cross Research S.A., Arzo, \\ Switzerland, ${ }^{4}$ Abiogen Pharma S.p.A., Pisa, Italy
}

In a double-blind, placebo-controlled, multiple-ascending-dose study, the encephalotropic and psychotropic properties of ABIO$08 / 01$, a new isoxazoline, were studied in 16 young healthy males. In the randomized non-balanced phase- 1 study, they received 3 oral drug doses $(10,20,40 \mathrm{mg})$ and placebo for 7 days (wash-out period 8 days). EEG recordings and psychometry were carried out at the hours $0,1,6$ of day 1 (acute effect) and day 5 (subacute and superimposed effects). Auditory ERPs were recorded at the 
hours 0 and 2 of days 1 and 5. Descriptive data analysis revealed a dose- and baseline-dependent EEG-effect: activating patterns in the resting-EEG (40 mg $>20 \mathrm{mg}>10 \mathrm{mg}$ ) and for the high dose in the vigilance-controlled EEG, and sedative patterns for the low dose in the vigilance-controlled EEG and in the eyes-open-EEG (10 mg > $20 \mathrm{mg}>40 \mathrm{mg}$ ). Confirmatory statistics on P300 latency demonstrated a significant shortening after acute, subacute and superimposed administration of $40 \mathrm{mg}$, suggesting accelerated cognitive information processing speed. While ERP amplitudes showed only minor effects, low-resolution brain electromagnetic tomography (LORETA) demonstrated that ABIO-08/01 promotes more efficient information processing by re-allocating perceptual and cognitive ERP resources. EEG tomography revealed hemisphere-specific changes (increases left temporo-parietal and decreases right prefrontal) which are in accordance with neuroimaging studies in anxiety and our previous LORETA findings with buspirone. Thus, our pharmaco-EEG/ERP studies confirm early pharmacological findings in animals of a cognition-enhancing effect of ABIO-08/01, which is interesting in the context of the anxiolytic mode of action of the compound as its CNS effects are quite different from those of anxiolytic sedatives, such as benzodiazepines.

\section{Free Oral Communications}

01

\section{A Regression Approach of Inter EEG Signals Coherence - Method and Usefulness}

\section{Sebban, C. Debouzy, V.H. Nguyen-Michel}

Hôpital Charles Foix, Unité d'Explorations Fonctionnelles, Ivry sur Seine, France

Coherence between two signals evaluates their time dependency which results mainly from the inter signals phase lag stability. Generality coherence is expressed as an unsigned correlation coefficient. In our system, we apply a regression approach of the coherence. This method perfectly correct from a mathematical point of view, splits on. The activity on site A in two parts according to the activity of another site (B). The first part is totally dependant on $\mathrm{B}$, the second totally independent. We can compute the phase lag for the first part. Dividing the first part by the total activity in A gives the coherence ratio. The most important is that variations of both parts induced by the same stimulus (physical or pharmacological) can be evaluated separately. This is useful for the diagnosis of dementia in aged patients as well as for pharmacological studies. For example, diazepam effects in Pre Frontal cortex shows a better reproducibility when examined only on PFC activities independent of dorsal hippocampal EEG. Another step in this approach is to subtract from the activities on A those which are totally dependent with another signal. We have called this method "De-Coherence ». It allows a very good way to suppress artifacts. For example extraction of the part dependent on eyes movements from frontal activities can be easily studied which is particularly useful to evaluate frontal dysfunction in dementia.

\section{2 \\ Dose-Response Effects of Alcohol on Lane Keeping and P3a Amplitude During Simulated Driving}

A.E. Wester, K.B.E. Böcker, J.C. Verster, E.R. Volkerts, J.L. Kenemans

Dept. of Psychopharmacology, UIPS, RMI, Utrecht, The

Netherlands

Recently we published an integrated ERP - simulated driving test for studying attention during driving. In this test $\mathrm{P} 3 \mathrm{a}$ is evoked by presenting an oddball series with $80 \%$ standards, $10 \%$ deviants and $10 \%$ novel sounds during simulated driving. The aim of the present study was to assess the effects of alcohol on lane keeping, exogenous attention capture, as assessed by P3a amplitude, and their possible relationship. To that end 30 subjects performed the primary DASS simulated driving task with Blood Alcohol Concentrations of 0.0, 0.2, 0.5, 0.8 and $1.0 \%$ in a double-blind cross-over design. During parts of this task a secondary oddball series was presented and ERPs were recorded. Lane keeping and P3a amplitudes were analyzed for alcohol effects, both when recorded in isolation (single task) and simultaneously (dual task). As expected lane keeping got worse above $0.2 \%$ BAC ( $<<$ $.001)$, even more so in the dual task condition $(\mathrm{p}<.05)$. Similarly, P3a decreased above $0.2 \%$ BAC $(\mathrm{p}<.01)$, in the dual as well as the single task condition. From the decrease in P3a it was concluded that alcohol diminished the attentional resources available for exogenous capture. This decrease in attentional resources may also have worsened lane keeping. However, the effects of alcohol on P3a and lane keeping were not correlated. The data will be further discussed with respect to dose-response effects on other perceptual and attentional processes as indicated by other ERP components (such as N1, mismatch negativity, P3b) and their possible relations to lane keeping. This will provide further insight in the sensitivity of the integrated ERP - simulated driving test and possibilities for its future use in testing drugs for driving safety.

03

\section{Etomidate Activates Irritative Areas in Patients with Temporal Lobe Epilepsy}

R. Wix ${ }^{1}$, M.L.Meilán' ${ }^{2}$ J.L. Martínez-Chacón ${ }^{2}$,E. de Dios' ${ }^{2}$, R.G. Sola², J. Pastor ${ }^{1}$

${ }^{1}$ Clinical Neurophysiology, ${ }^{2}$ Anaesthesiology and Reanimation, ${ }^{3}$ Neurosurgery, Epilepsy Unit, Hospital Universitario La Princesa, Madrid, Spain

Purpose: To analyze the changes induced by etomidate (Et) in the EEG and temporal mesial areas recorded with foramen ovale electrodes (FOE). Methods: Eighteen patients (11 men and 7 women), ages ranging 22 - 52 years, suffering from tempo- 
ral lobe epilepsy (TLE) were evaluated. Et was i.v. perfused (0.1 $\mathrm{mg} / \mathrm{kg}$ ) meanwhile EEG (scalp and FOE), heart rate, EKG and $\mathrm{SaO} 2$ were monitored. Basal activities were defined as five minutes prior Et perfusion. The onset seizure (OS) zone was defined from $\mathrm{v}$-EEG+FOE in a double-blind method. The frequency (spikes/min) induced by $\mathrm{Et}$ in mesial and lateral regions were measured. In mesial recordings, for every minute (n), we defined $\Delta(\mathrm{n})=$ freqleft $(\mathrm{n})$-freqrigth $(\mathrm{n})$, and calculate the mean $(\mathrm{v})$ and SD for basal activity. Confidence limits were either 3SD. The epileptogenic zone was defined as right if $\Delta>v+3$ SD for some $\mathrm{n} \geq$ 1 , as left if $\Delta<v-3 S D$, for some $n \geq 1$, or indifferent in other case. Results: 1$)$ Side effects were mild distal myoclonias (12/18), severe myoclonias arm $(2 / 18)$, pain during infusion $(3 / 18)$ and euphoric reaction $(1 / 18) ; 2)$ Heart rate, breath rate and $\mathrm{SaO} 2$ did not change with Et; 3) The frequency of the spikes increased in OS area from the first minute and persisted above the basal level until at least $14 \mathrm{~min}$; 4) The frequency of the spikes followed simple exponential rise and decay; 5) Et lateralized correctly the OS lobe in 18/18 patients with FOE recordings and 3/15 with scalp recordings. Conclusions: Et perfusion is an easy, safe and accurate method for identify the OS in TLE patients.

This work has been financed by, Ministerio de Sanidad FIS PI06/0349.

\section{4}

Mean Lobe Intracortical Distribution of Electrical Activity after Benzodiazepine Intake: A Dose-Range Study

M.J. Barbanoj 1,2, S. Romero ${ }^{1,2}$, R.M. Antonijoan' ${ }^{1}$, J. Riba

${ }^{1} \mathrm{CIM}$-Sant Pau, IR, Servei de Farmacologia Clínica, HSCSP, Barcelona, Spain; ${ }^{2}$ Departament de Farmacologia i Terapèutica, UAB, Barcelona, Spain; ${ }^{3}$ Centre de Recerca en Enginyeria Biomèdica, Departament ESAll, UPC, Barcelona, Spain

Background: LORETA algorithm allows the computation of intracerebral measures of neuroelectrical activity from the scalp EEG data. It computes the 3-D distribution of current densities at each voxel (6.239) for specified EEG frequency bands. Objective: To describe the time-course of mean lobe intracortical power of current densities after three increasing oral doses of a benzodiazepine compound. Methods: Nine healthy male volunteers participated in a randomised, double-blind, cross-over, placebocontrolled explorative study to assess the effects of single oral doses of alprazolam $0.25,0.5$ and $1 \mathrm{mg}$. Three minutes eyes closed V-EEG were recorded from 19 leads (plus V-EOG and H-EOG) at PRE, $+30,+45,+60,+90,+150$ and +240 minutes from drug administration. Mean spectra from six 5-second artefact-free epochs was computed, evaluating alpha- $1(7.5-10.5 \mathrm{~Hz})$ and beta- 1 $(13-16 \mathrm{~Hz})$. Standardized LORETA was employed to calculate mean lobe intracortical power of current densities. Net drug effects were computed as differences from PRE and from PLACEBO. Intrasubject 3-way (lobe, dose, time) GLMs comparing between pair of lobes were applied to each frequency band separately. Results: The biggest changes (decreases in alpha-1 and increases in beta-1) were observed in both frequency bands at the parietal lobe. The smallest changes were obtained at the frontal lobe in alpha- 1 and at the temporal lobe in beta-1. Changes observed at the parietal lobe were significantly different from those observed at the limbic, sublobar and frontal lobes in alpha-1 and the sublobar and temporal lobes in beta-1. In addition, alpha-1 decreases at limbic, sublobar and frontal lobes were significantly lower than those observed at occipital and temporal lobes and those at the temporal lower than at the occipital lobe. Moreover, only beta- 1 increases at temporal were significantly lower than those observed at the limbic lobe. Conclusions: Changes on intracortical power of current densities after increasing oral doses of alprazolam in healthy male volunteers were different as a function of lobe and frequency band. Decreases in alpha-1 showed more significant inter-lobe differences than increases in beta- 1 .

The research team from CIM-Sant Pau is member of CIBERSAM (supported by Spanish Ministry of Health, Instituto de Salud Carlos III).

05

QEEG- Neurometric Analysis Guided Neurofeedback
(NF) Treatment in Medicated Schizophrenia Patients.
Case Study: 53 Patients: How Neurometric Analysis is
Important at the Treatment of Schizophrenia as well
as Diagnosis?

T. Sürmeli

Living Health Center for Research and Education, İstanbul, Turkey

Introduction: EEG studies of schizophrenic patients indicate a higher number of patients with abnormal records decreased in alpha activity, increased delta and theta activity and possibly more left sided abnormalities, and some coherence abnormalities. There is no cure, it is treatable with antipsychotic drugs. It shows an enormous need for developing better methods of delivering treatments and better ways to manage the disease. We thought neurofeedback treatment may address positive symptoms, negative symptoms, cognitive dysfunction, nonadherence to treatment while the patient is on or not on antypsychotics. Methods: Most of the patients were diagnosed with chronic schizophrenia before coming to our center and did not improve in their symptoms with antipsychotic medications. Neurometric analysis of the QEEG of the patients suggested chronic schizophrenia consistent with the clinical judgement of the author. All 52 patients received QEEG analysis with the Nxlink data base, PANSS(Positive and Negative Syndrome Scale) at the baseline and at the end of the treatment..31 out of 52 patients also received TOVA and MMPI at baseline and at the end of the treatment. Responses to PANSS was were analysed to evaluate the effectiveness of neurofeedback. All patients received between 40-120 neurofeedback training sessions. NF protocols were chosen according to their QEEG neurometric analysis. Results: 47 schizophrenic patients improved after neurofeedback treatment. 18 schizophrenic patients who received neurofeedback training showed significant improvement based on Nxlink, and PANSS. 29 out of 31 schizophrenic patients who received neurofeedback training showed significant improvement 
based on QEEG analysis with Nxlink data base, PANSS as well as the TOVA and MMPI results. 3 out of 52 patients dropped the treatment. 2 out of 52 did not respond. The patients who showed coherence abnormalities in the QEEG and who can normalize the coherence abnormality responded better and quicker to neurofeedback training. Antipsychotic medications may increase coherence abnormalities in the brain but coherence abnormalities can be corrected or diminished with neurofeedback treatment to get a better outcome. NF treatment may increase adherence to treatment and reduce possible side effects of antipsychotic in schizophrenia. Further study with controls is warranted.

\section{6}

\section{Effect of Subanesthetic Dose of Ketamine on the Brain Activity in Model of Schizophrenia I: A} Standardized Low-Resolution Brain Electromagnetic Tomography (sLORETA) Study

\section{Brunovsky, J. Horacek, B. Tislerova, P. Sos, T. Novak, V. Krajca}

Prague Psychiatric Center,Prague, Czech Republic; Dept. of Neurology, Faculty Hospital Bulovka, Prague, Czech Republic

Rationale: Ketamine, a noncompetitive N-methyl-D-aspartate antagonist, is known to induce a broad range of psychopathological symptoms similar to those seen in schizophrenic patients. In randomized placebo-controlled trial we studied effects of ketamine on cortical EEG sources in healthy volunteers. Methods: EEG recordings were obtained from 20 healthy subjects during resting condition. Ketamine (or placebo) was applied i.v. in the dose of $0.27 \mathrm{mg} / \mathrm{kg}$ within first $10 \mathrm{~min}$., followed by a maintenance infusion of $0.27 \mathrm{mg} / \mathrm{kg} / \mathrm{h}$ for $20 \mathrm{~min}$. The intracerebral current density distribution was computed from spectrally analyzed data (at baseline and 10 and $20 \mathrm{~min}$ after administration) by means of standardized low-resolution brain electromagnetic tomography (sLORETA). The localization of the differences in source distribution was assessed by voxel-by-voxel paired t-tests of sLORETA images of the log-transformed current density power in eight frequency bands. Results: Statistically significant differences were observed for sLORETA power both 10 and 20 min after dosing, with decrease of magnitude of alpha- 1 and alpha- 2 sources over large posterior regions as well as decrease of magnitude of beta- 1 and beta- 2 sources in the posterior cingulate and precuneus. Cortical beta-3 and gamma sources significantly increased mainly in the cingulate and parahippocampal structures and over the right fronto-temporo-parietal cortical regions. Conclusion: Our results suggest the involvement of heteromodal association cortex and limbic structures in the psychological and neurophysiological effects elicited by ketamine.

This work was supported by the grant No.NR/9330-3 (IGA MZ CR).
07

\section{Spectral Power Density in the Early Course of Schizophrenia}

M.O. Pflueger ${ }^{1}$, U. Gschwandtner ${ }^{1}$, R. Zimmermann ${ }^{1}$, C. Schindler ${ }^{2}$, A. Riecher ${ }^{1}$, P. Fuhr ${ }^{3}$

${ }^{1}$ Psychiatric Outpatient Department, ${ }^{2}$ Institute of Social and Preventive Medicine, ${ }^{3}$ Department of Neurology, University Hospital Basel, Switzerland

Background: There is evidence for a drop in alpha2 and an excess in delta/theta power in patients with schizophrenia as compared with healthy controls (HC). This might be related to vulnerability or course of the disease. However, it is unclear whether this pattern of abnormal spectral power density is present already in the first episode (FE) or even in an at risk mental state (ARMS) for psychosis. Methods: EEG of $40 \mathrm{FE}, 37$ ARMS subjects and $24 \mathrm{HC}$ were recorded under resting conditions with eyes closed. Fast Fourier Transform was performed. The power spectrum was subdivided into 7 bands. A Mixed Model Analysis was done with individuals as random factor, group and electrode as independent factors, and log band power as dependant factor. Results: There was a significant group main effect in the alpha2 (F1,62 $=5.29, \mathrm{p}$ $=0.025)$, theta $(\mathrm{F} 1,62=9.61, \mathrm{p}=0.03)$ and delta $(\mathrm{F} 1,62=4.99, \mathrm{p}=$ $0.029)$ power density reflecting a higher power in $\mathrm{HC}$ than in $\mathrm{FE}$ patients. In general, power density in posterior parts of the brain was larger than in anterior parts as shown by significant Helmert contrasts according to mean power ordered electrodes. The inclusion of ARMS individuals diminished the overall group effect since the ARMS individuals power almost perfectly fell into an intermediate range between FE and HC. Conclusion: The classical pattern of spectral power in delta, theta and alpha2 has been partially replicated with alpha2 power decrease in FE. The findings in delta/theta power contradict earlier results. The additional consideration of ARMS subjects suggests that the observed pattern is stable and valid particularly in the prodromal state of schizophrenia. Therefore, we propose the alpha2/delta/theta power density pattern to reflect a state-dependant alpha2 decrease while delta/ theta increase might reflect chronicity.

\section{8}

\section{EEG in the Detection of the Beginning Psychosis}

U. Gschwandtner ${ }^{1}$, M.O. Pflueger ${ }^{1}$, M. Gaggiotti ${ }^{2}$, A. Riecher ${ }^{1}$, P. Fuhr ${ }^{2}$

${ }^{1}$ Psychiatric Outpatient Department, ${ }^{2}$ Department of Neurology, University Hospital, Basel, Switzerland

Background: EEG investigation in patients with an 'At Risk Mental State' (ARMS) for psychosis and patients with a first episode of psychosis (FE) in comparison to healthy controls (HC) in a clinical follow up study of Early Detection of Psychosis. Methods: Seventy-three patients (42 ARMS, $31 \mathrm{FE)} \mathrm{and} 35 \mathrm{HC}$ were investigated. ARMS patients were followed up in order to monitor transition to psychosis. Psychopathology was assessed with respect 
to positive and negative symptoms. At study baseline EEG was recorded using the 10/20 system. Two blinded neurologists analyzed the EEGs visually for presence of generalized or focal slowing and epileptiform discharges. EEG data were controlled for medication and substance abuse. For statistical analyses we used Chi2-tests, logistic regression, ANOVA, and receiver operating characteristics (ROC). Results: Patients showed significantly more pathological EEG abnormalities located more frequently in temporal or fronto-temporal regions $(\mathrm{P}<0.01)$ of the brain than healthy controls $(P<0.05)$, with twice as many pathologies in ARMS than in FE patients. A pathological EEG was significantly associated with a decreased risk for transition to psychosis. The predictive specificity of psychosis could be enhanced from $59 \%$ to $73 \%$ by considering EEG pathology in addition to psychopathology alone. Conclusion: These results show that EEG investigation in patients suspected for psychosis can be supportive in the identification of patients who will develop psychosis later on.
09

\section{QEEG Parameters as Indicator for Negative Symptoms in Patients with First Episode Schizophrenia}

\author{
R. Zimmermann ${ }^{1}$, U. Gschwandtner ${ }^{1}$, M.O. Pflueger ${ }^{1}$, A. Riecher ${ }^{1}$ \\ P. Fuhr ${ }^{2}$
}

${ }^{1}$ Psychiatric Outpatient Department and ${ }^{2}$ Department of Neurology, University Hospital, Basel, Switzerland

Background: While several studies showed an association of QEEG band power with negative symptoms in patients with schizophrenia this has not been investigated in neurolepticnaïve first episode patients (NNFE) up to now. We hypothesised delta $(0.5-4 \mathrm{~Hz})$ and theta $(4-8 \mathrm{~Hz})$ power to be augmented and alpha power to be diminished with increased negative symptoms in NNFE. We expected no relationship of positive symptoms and EEG band power. Methods: We investigated 27 NNFE. Psychopathology was rated with Scale for the Assessment of Negative Symptoms (SANS) and the Brief Psychotic Rating Scale (BPRS). EEG was recorded from 8 electrodes with 10/20 system. After artefact removal a Fast Fourier Transform was performed across 7 frequency bands. Linear regressions with log transformed power as dependent, psychopathology as independent and medication, drugs, age, sex, education, day time of EEG recording as confounding factors were calculated. Results: A significant positive correlation of SANS with delta $(\mathrm{p}=.04)$ and theta $(\mathrm{p}=.03)$ could be replicated in NNFE. The negative correlation of alpha power density with SANS scores could not be confirmed. Interestingly, we found betal $(12-15 \mathrm{~Hz})$ to be positively correlated with negative syndrome scale. As expected no relationship of BPRS summary score and EEG power could be found. Conclusion: Former studies of QEEG in schizophrenic patients could be partially replicated in NNFE. Correlation of negative symptomatic with power of low frequency bands could be confirmed. While reduction of power of alpha bands observed in FE (Pflueger et al., 2008), there is no correlation with sans. Recording EEG in beginning psychosis may therefore be helpful in the diagnosis of a beginning negative syndrome in schizophrenia. 
Ahnaou, A. S1.2

Aiba, S. P8

Anderer, P. P14, S3.1

Anderson, K. S1.1

Antonijevic, I.A. S4.4

Antonijoan, R.M. O4

Arns, M. S6.1, S6.4

Assandri, A. P14

Babiloni, C. S2.2

Babiloni, F. S8.2

Barbanoj, M.J. S8.1, S8.4, O4

Bares, M. P12

Barnett, K.J. S6.2

Bartos, A. P2

Battaglia, F.P. S5.2

Baumann, K. S4.2

Beck, J. S4.3

Benchenane, K. S5.2

Benz, M. S7.2

Böcker, K.B.E. O2

Boeijinga, P.H. P5, P6

Boikova, T.V. P11

Bootsma, A. S6.4

Brand, S. S4.2

Breteler, M. S6.1

Brunovsky, M. P1, P2, P4, P12, O6

Bubenikova-Valesova, V. P4

Christian, E.P. S1.4

Cook, I.A. S3.2

Debouzy, C. O1

De Carli, C. S2.2

Del Percio, C. S2.2

Dezortova, M. P1

Diblikova, F. P2

Dierks, T. S3.3, S3.4

Dios, E. de O3

Dodel, R. S4.5

Doherty, J.J. S1.4

Drinkenburg, W.H.I.M. S1.2

Duncan, G. S7.2

Elands, C. S1.1

Eusebi, F. S2.2

Fitzgerald, P. S6.4

Fokin, V.F. P10

Frisoni Giovanni, B. S2.2

Fuhr, P. O7, O8, O9

Fujakova, M. P4
Gaetani, S. S5.1

Gaggiotti, M. O8

Gaudias, J. S7.3

Geroldi, C. S2.2

Giesler, M. S4.5

Gilmer, W.S. S3.2

Gispen-de Wied, C.C S7.1

Grasa, E. S8.4

Greenwald, S.D. S3.2

Gschwandtner, U. O7, O8, O9

Gunkelman, J. S6.1

Haag, A. S4.5

Hatzinger, M. S4.2, S4.3

Hell, D. S7.2

Hemmeter, U. S4.5

Hemmeter, U.M. S4.2, S4.3

Higashima, M. S3.4

Hirata, K. S2.3, P7, P8, P9

Hoffman, D.A. S6.3

Holsboer-Trachsler, E. S4.2, S4.3

Horacek, J. P1, P4, O6

Hoshino, Y. S2.3, P7, P9

Howland, R.H. S3.2

Isenhart, R. S2.3

Ising, M. S4.2

Ivashtenko, E.I. P10

Iwanami, H. P8

Jay, T.M. S5.3

John, E.R. S2.3

Kahkonen, S. S7.4

Kahn, R.S. S7.1

Kemner, C. S7.1

Kenemans, J.L. O2

Khamassi, M. S5.2

Kicic, D. S7.4

Kikuchi, M. S3.3, S3.4

Kimoto, K. P8, P9

Kimura, M. S4.1

Kluge, M. S4.4

Koenig, T. S3.3, S3.4

Komagamine, T. P8

Kometer, M. P3

Konovalov, R.N. P10

Kopecek, M. P12

Korostenskaja, M. S7.4

Korovaiceva, G.I. P11

Koshino, Y. S3.4

Kraca, V. P12
Krajca, V. P2, O6

Krieg, J.C. S4.5

Krotenkova, M.V. P10

Kutova, M. P4

Kuznetsova, G.D. S5.1

Lantz, G. S2.4

Larsson Pål, G. S2.4

Lehmann, D. S3.3

Lengellé, R. P5

Leuchter, A.F. S3.2

Luthringer, R. P6

Marston, H.M. S1.1

Martínez-Chacón, J.L. O3

Meilán, M.L. O3

Michel, C.M. S2.4

Midzyanovskaya, I. S5.1

Muheim, F. S4.3

Murck, H. S4.4

Nannipieri, F. P14

Nessi, P. P14

Ngomba, R. S5.1

Nguyen-Michel, V.H. O1

Nikulin, V.V. S7.4

Nikulina, A.V. S7.4

Nikzad, R. S3.1

Novak, T. P1, P12, O6

Ogawa, T. P7, P8, P9

Okabe, R. P8

Oranje, B. S7.1

Osawa, M. S2.1

Palenicek, T. P4

Pascual-Marqui, R.D. S2.3

Pastor, J. O3

Penzel, T. S4.5

Peyrache, A. S5.2

Pflueger, M.O. O7, O8, O9

Philippens, I.H.C.H.M. S1.3

Pievani, M. S2.2

Pinault, D. S7.3

Pintzinger, N. S3.1

Ponomareva, N.V. P10, P11

Potts, B. S1.4

Prichep, L. S2.3

Quirk, M.C. S1.4

Riba, J. O4, S8.1, S8.3

\section{KARGER}

(c) 2009 S. Karger AG, Basel

Fax +4161306 1234

E-Mail karger@karger.ch

www.karger.com 
Riecher, A. O7, O8, O9

Rocamora, R. S4.5

Rogaev, E.I. P11

Romero, S. O4, S8.1

Rosini, S. P14

Rossini, P.M. S2.2

Ruigt, G.S.F. S1.1

Saletu, B. S3.1, P14

Saletu-Zyhlarz, G.M. S3.1, P14

Schindler, C. O7

Schüssler, P. S4.4

Sebban, C. S5.3, O1

Seeck, M. S2.4

Semlitsch, H.V. P14

Snyder, D.H. S1.4

Sola, R.G. O3

Song, W. S1.4

Sos, P. P4, P12, O6

Soufflet, L. P6

Spaniel, F. P1

Spedding, M. S5.3

Spronk, D. S6.1, S6.4

Stanek, J. S3.1
Steiger, A. S4.4

Stone, B.M. P13

Strik, W. S3.3, S3.4

Sürmeli, T. O5

Tajmir-Riahi, A. S3.1

Takashima, R. P7, P8, P9

Tanaka, H. S2.3, P7, P8, P9

Thum, A. S4.5

Tintera, J. P1

Tislerova, B. P1, P4, P12, O6

Tohmé, M. P5

Tolmacheva, E. S7.3

Treyer, V. S7.2

Trivedi, M.H. S3.2

Turner, C. P13

Valle, M. S8.4

Van Rijn, C.M. S5.1

Van Ruth, R. S6.4

Vanwersch, R.A.P. S1.3

Vecchio, F. S2.2

Verbaten, M.N. S7.1

Verhave, P.S. S1.3
Verster, J.C. O2

Vijn, P.C.M. S1.1

Volkerts, E.R. O2

Vollenweider, F.X. S7.2, P3

Vysata, O. P2

Wada, Y. S3.4

Ward, N. S1.1

Watanabe, Y. P7, P8, P9

Westenberg, H.G.M. S7.1

Wester, A.E. O2

Wiener, S.I. S5.2

Wix, R. O3

Wolzt, M. P14

Wright, N.A. P13

Yoshimura, M. S3.3

Zheng, T. S7.3

Zimmermann, R. O7, O9 